NISSUNA UMANA INVESTIGAZIONE SI PUO DIMANDARE VERA SCIENZIA S'ESSA NON PASSA PER LE MATEMATICHE DIMOSTRAZIONI LEONARDO DA VINCI

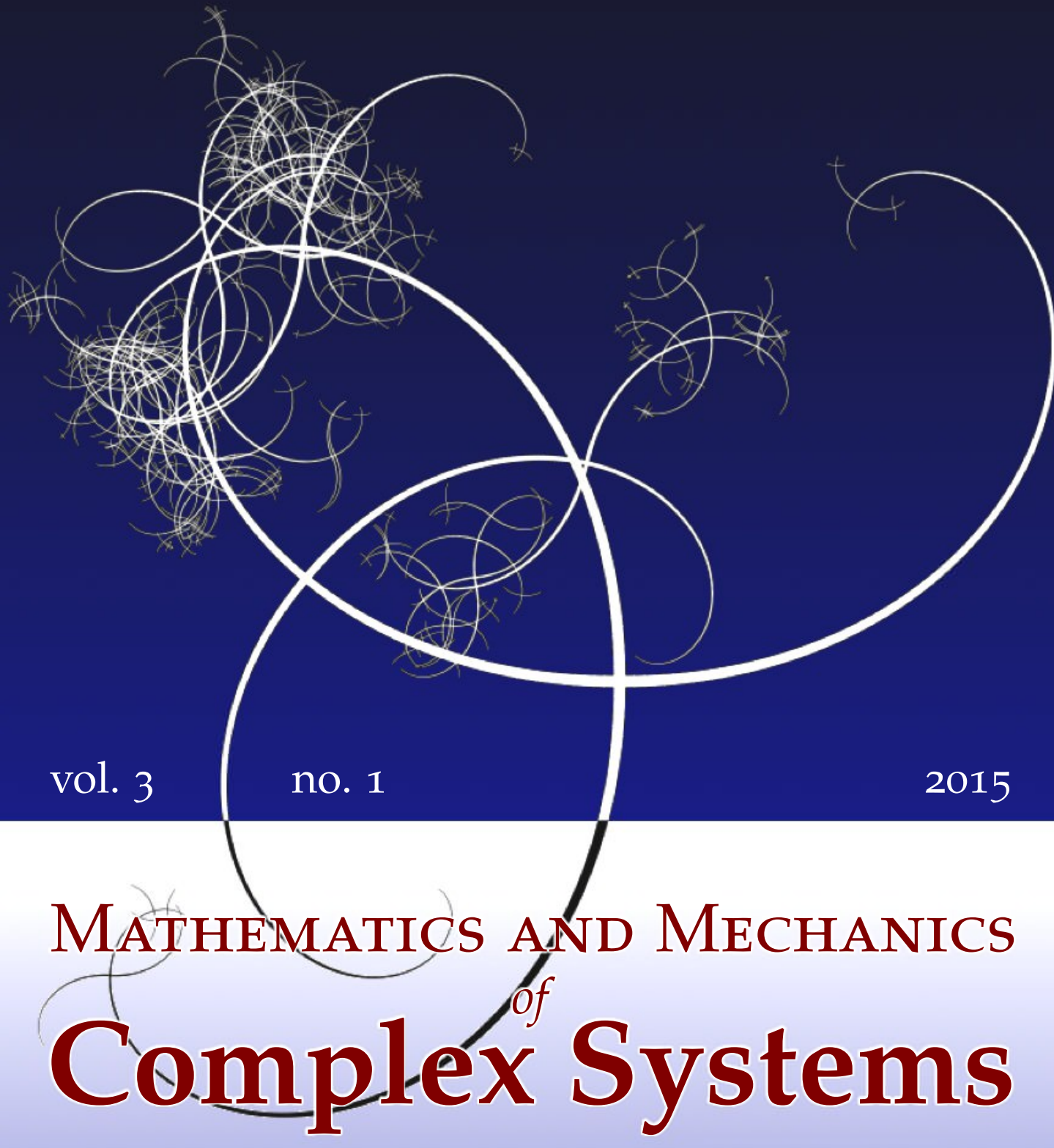

JÜRI ENGELBRECHT AND ARKADI BEREZOVSKI

REFLECTIONS ON

MATHEMATICAL MODELS OF DEFORMATION WAVES

IN ELASTIC MICROSTRUCTURED SOLIDS 


\title{
REFLECTIONS ON \\ MATHEMATICAL MODELS OF DEFORMATION WAVES IN ELASTIC MICROSTRUCTURED SOLIDS
}

\author{
JÜRI ENGELBRECHT AND ARKADI BEREZOVSKI
}

\begin{abstract}
This paper describes the mathematical models derived for wave propagation in solids with internal structure. The focus of the overview is on one-dimensional models which enlarge the classical wave equation by higher-order terms. The crucial parameter in models is the ratio of characteristic lengths of the excitation and the internal structure. Novel approaches based on the concept of internal variables permit one to take the thermodynamical conditions into account directly. Examples of generalisations include frequency-dependent multiscale models, nonlinear models and thermoelasticity. The substructural complexity within the framework of elasticity gives rise to dispersion of waves. Dispersion analysis shows that acoustic and optical branches of dispersion curves together describe properly wave phenomena in microstructured solids. In the case of nonlinear models, the governing equations are of the Boussinesq type. It is argued that such models of waves in solids with microstructure display properties that can be analysed as phenomena of complexity.
\end{abstract}

\section{Introduction}

Waves are not only carriers of energy; they are also carriers of information. This means that waves generated by certain initial and boundary conditions carry the information not only from those conditions, but the information about the properties of the media they meet in their course of propagation as well. This information is reflected in changes of the wave profiles or, in other words, in changes of their spectra. In the present overview we focus on the influence of microstructure of solids on the macrobehaviour of deformation waves. Although deformation waves in solids have been studied for over a century, new and intriguing applications continue to arise in contemporary engineering problems. Solids with unconventional

\section{Communicated by Francesco dell'Isola.}

This research was supported by the EU through the European Regional Development Fund and by the Estonian Ministry of Education and Research (SF 0140077s08). The authors would like to thank all their coauthors and colleagues for valuable discussions.

MSC2010: 74A30, 74J99, 74E05, 74H99.

Keywords: wave motion, microstructured solids, internal variables, dispersion, nonlinearity. 
properties resulting from their composition and internal architecture and their wave propagation characteristics will be of interest along with the novel technologies that they inspire. Of particular interest are multiscale wave processes in solids characterised by hierarchical structures, with nested levels of geometric and/or material complexity, as well as multiphysics phenomena due to coupled mechanical, thermal and electromagnetic effects. Besides, deformation waves can be regarded as attractive characterisation tools, due to their sensitivity to small-scale structural features, such as discontinuities, interphases and defects.

Every material body has actually a microstructure at a smaller scale. Take for example alloys, polycrystalline solids, ceramic composites, functionally graded materials, granular materials, etc. From this viewpoint, there exists an intrinsic space-scale which should be taken into account in deriving the governing equation of motion. The natural question is: when is it needed? Clearly one should pay attention to two characteristic lengths: the characteristic length $L_{0}$ of an external excitation (the wavelength) and the internal characteristic length $l$. When $L_{0} / l \geq 1$, the conventional theories and corresponding mathematical models can be effectively used because the microstructure acts collaboratively. When $L_{0} / l \approx 1$, the influence of a microstructure becomes important, which demonstrates the nonlocality of the wave propagation [Engelbrecht and Braun 1998]. In terms of time-scale, the highfrequency excitations (corresponding to short wavelengths) should also strongly be influenced by the presence of the microstructure. All this calls for more sophisticated and physically well-grounded modelling where the conventional assumptions for constructing the theories might not work, and attention should be focussed on catching effects caused by the internal structure of materials. Although there are many theoretical studies in this field, the space-scale in real dimensions is not always introduced. That is why some estimates should here be given. The internal characteristic lengths certainly vary largely in scale [Gates et al. 2005]. If we take a characteristic size of a structural element of $10^{0} \mathrm{~m}$, then in typical materials the characteristic internal lengths are between $10^{-3} \mathrm{~m}$ and $10^{-6} \mathrm{~m}$. However, in concrete, for example, the internal lengths can be around $10^{-2} \mathrm{~m}$. We leave aside here smaller and larger internal lengths like in nanostructured materials (in $\mathrm{nm}$ ) or seismology (in km).

As mentioned by Eringen [1999], "the published work in microcontinuum mechanics is so large" that we do not aim to present an overwhelming review, but shall concentrate our attention on the mathematical modelling of elastic deformation waves. Even more, in order to explain the various models, we limit ourselves to the analysis of one-dimensional longitudinal waves as a benchmark, although in most cases the more general three-dimensional theories exist as a basis for deriving them. This gives a possibility to compare the interaction effects between macroand microstructure in a simple and transparent manner and find unified patterns. 
In addition, it gives a possibility to analyse the changes which occur to one of the three cornerstones of mathematical physics - to the classical wave equation which will turn into an equation with higher-order terms governing the dispersion effects. Some basic concepts should be reviewed in order to explain descriptions used in various studies:

- Microstructured solids display "material substructural complexity" [Mariano and Stazi 2005] because of interaction between their constituents.

- Substructural complexity can be characterised as "complexity of particles" [Kröner 1968], which leads to nonlocality and wave dispersion.

- In mathematical models for waves, higher-order spatial gradients should be accompanied by higher-order time derivatives [Metrikine 2006] and be dynamically consistent [Askes and Aifantis 2006].

An overview is needed for summing up the previous research on models of waves in microstructured solids and demonstrating their essential features. The present overview is initiated by many others, which sometimes are selective, emphasising the author's sympathies. We tried to pay due attention to all essential results, to the best of our knowledge.

The paper is organised as follows. In Section 2, a brief description of basic theories is presented, including discrete and continuum theories and links between them. This is the basis for Section 3, where various one-dimensional mathematical models are presented and compared. Section 4 is devoted to the generalisation of models involving multiscale and multifield cases, and in Sections 5 to 7 the analysis of physical effects observed in the macro- and microbehaviour of waves in microstructured solids is presented. Finally, in Section 8 a summary of results and further discussion are given.

\section{Theoretical landscape of modelling}

The idea of theories relies on the mathematical modelling of phenomena under consideration. This was already known to Leonardo da Vinci: observe the phenomenon, and list quantities having numerical magnitude that seem to influence it. Although the great master recommended setting up linear relations among the pairs of these quantities, it was a considerable step forward to contemporary physical sciences. Nowadays we certainly know about the importance of being nonlinear, but in many cases the basic concepts follow the advice of Leonardo da Vinci, as will be explicitly seen from what follows.

In the case of microstructured solids, the deformation waves, as said in Section 1, are influenced by the microstructure. In an ideal case, the behaviour of materials should be simulated, with only the constitutive law describing interactions between 
atoms. However, explicit calculation of all of the atomic degrees of freedom will never be feasible due to the scales of deformation that are important in realistic problems. The only possibility is to selectively remove most of these degrees of freedom to make the problem tractable [Curtin and Miller 2003]. Thus, the crucial point in modelling wave phenomena is to choose the starting point: either to start from a discrete (lattice structure) or from a continuous (continuum) model in order to capture size effects of the microstructure. Here one should remember that "mass point and continuum theories have equal rights in the classical mechanics of matter" [Kröner 1968].

So, attention should be paid to (i) discrete models (bottom-up approach); (ii) continuum models (top-down approach); and (iii) links between discrete and continuum models.

2.1. Discrete models. A discrete model takes into account all the constituents of a solid by treating them as point-like masses. For example, the monocrystalline solids have a regular arrangement of their constituents; in polycrystalline solids the arrangements are more complicated [Maugin 1999]. We leave aside here amorphous materials and liquid crystals. The most intriguing question is how to establish (postulate) the interactive forces between the constituents which are included into the equations of motion for every point-like mass (usually Newton's law).

The well-known studies of Brillouin [1946] and Askar [1986] have analysed the basic cases, including diatomic and polyatomic chains. Kunin [1975] has derived the governing equations for a diatomic chain with an additional average relative deformation (called microdeformation) of the elementary cell together with a deep mathematical analysis of corresponding models. Maugin [1999] has presented a contemporary description of waves in elastic crystals (lattice dynamics).

Probably, the best-known discrete model for waves in a one-dimensional lattice is the Born-von Kármán model [1912], (see also [Maugin 1999]). This model and its counterparts for more complicated cases form the basis for the derivation of the higher-order dispersive wave equation by continualisation procedures; see for example [Maugin 1999; Askes and Metrikine 2005; Askes et al. 2008; Andrianov et al. 2010]. However, as remarked in [Seeger 2010], the explicit solution of the Born-von Kármán model derived by Schrödinger [1914] predicts that very distant particles start to move, even at arbitrarily small time after any localised perturbation. This physically inconsistent situation can be avoided by taking inertia of particles into account and using more complicated continualisation techniques. There are several studies along this line [Metrikine and Askes 2002; Askes and Aifantis 2006; Polyzos and Fotiadis 2012] resulting in strain-gradient models. This means actually following the principle of dynamic consistency [Metrikine 2006].

Besides one-dimensional chains, also two-dimensional structures are analysed; see [Maugin 1999; Pichugin et al. 2008; Andrianov et al. 2010]. The studies of 
granular media include also the modelling of granular lattices, i.e., chains of beads under Hertz contact [Coste et al. 1997; Porter et al. 2009].

2.2. Continuum models. Looking back in history, the important generalisation to account for the influence of a microstructure into the continuum theory was made by E. Cosserat and F. Cosserat [1909], who elaborated a theory with microrotations at each material point of a continuum. The degrees of freedom are then characterised by three rigid directors and the corresponding theory is nowadays called micropolar. If these directors are deformable, then the result is the micromorphic theory [Eringen and Suhubi 1964a; Eringen and Suhubi 1964b; Mindlin 1964; Eringen 1999]. When the directors are constrained in a special way (three microrotations and one microstretch), then the result is the microstretch theory [Eringen 1969]. Detailed descriptions of these theories can be found in the monographs [Eringen 1999; Capriz 1989; Maugin 2013]. In what follows we limit ourselves to one-dimensional micromorphic theory and leave aside the Cosserat-type microrotation. Analysis of the latter theory in the contemporary framework can be found in [Neff 2006; Neff and Jeong 2009].

Another avenue to generalisation is the inclusion of higher-order gradients of strain into the free energy function, as compared with the classical Cauchy theory, which accounts only for strains in constitutive equations; see [dell'Isola et al. 2009], for example. Such a possibility was pointed out already by Kröner [1968], and a detailed overview on gradient theories is given by Maugin [2013]. There are several studies on waves using this idea [Metrikine and Askes 2002; PapargyriBeskou et al. 2009; Polyzos and Fotiadis 2012]. A comparison of gradient and micromorphic theories is given by Kirchner and Steinmann [2004].

According to Kröner [1968], the complexity of particles in a solid plays a decisive role in deriving the mathematical models. This concept is explicitly used by Mindlin [1964], who has introduced a unit cell which "may also be interpreted as a molecule of a polymer, a crystallite of a polycrystal or a grain of a granular material". Such an approach is used in many later studies for deriving the governing equations of waves [Engelbrecht et al. 2005; Papargyri-Beskou et al. 2009; Porubov et al. 2009; Polyzos and Fotiadis 2012; Berezovski et al. 2013]. Mariano [2002] has used order parameters for describing, as he said, "the substructural configuration".

As far as our knowledge on internal structure of solids is not always explicitly described, one should think about replacing the physical structure by a certain field. Then the concept of internal variables has proved to be useful. Proposed originally to describe dissipative processes, and traced back to [Duhem 1911a; Duhem 1911b], the modern understanding is presented by Maugin and Muschik [1994]. The generalisation of this concept by introducing dual internal variables has made it possible to use for describing wave processes [Ván et al. 2008; Berezovski 
et al. 2011b]. This approach has a clear thermodynamic background, although the microstructure itself becomes latent and its influence can be considered as an additional field. Such a possibility is also mentioned by Capriz [1989] and Mariano [2002]. Germain [1973] has noted a possibility to introduce "hidden parameters" into the function of internal energy.

The separation of the macro- and microstructure in a continuum leads, in general terms, to the formulation of separate balance laws for each [Eringen and Suhubi 1964a; Eringen and Suhubi 1964b; Mindlin 1964; Eringen 1999; Mariano 2002]. Maugin [1993; 2011a] has proposed using the material formulation to represent the balance law of the macrostructure, which includes all the interaction forces within the solid, accounting for microstructural effects. Such an approach becomes very useful, when internal variables are introduced, for describing the effects due to the presence of the microstructure. Namely, the evolution of internal variables needed for determining the internal forces is then governed not by a balance law but by satisfying thermodynamical considerations [Ván et al. 2008; Berezovski et al. 2011b].

2.3. Links between lattice dynamics and continua. Calculation of values of continuum variables based on atomistic models has a long and rich history (see, for example, reviews by Goddard [1986], Zimmerman et al. [2002] and Webb et al. [2008]). Contemporary contributions include the thermomechanical equivalent continuum [Zhou 2005], generalised mathematical homogenisation method [Fish et al. 2005], and scale-dependent molecular averages [Murdoch 2010]. As concerns wave propagation, Chen et al. [2003; 2004] have justified applicability of microcontinuum theories from the atomistic viewpoint, and they stress the importance of size effects and phonon dispersion relation; see also [Chen and Lee 2003].

From the physical viewpoint, an extremely important question is how to establish the material parameters from discrete (atomic) models at the microscale. There are several studies in this direction based on experiments. Maranganti and Sharma [2007] have established strain-gradient elastic constants for various metals, semiconductors, silica and polymers by relating them to the atomic displacement correlations in a molecular dynamics computational ensemble (see also a long list of references on studies in this field). Zeng et al. [2006] have proposed to determine the dispersive elastic constants by using phonon dispersion relations. Dispersive phonon imaging is used by Jakata and Every [2008] for the cubic crystals $\mathrm{Ge}, \mathrm{Si}$, GaAs, and InSb.

Remark 1. We do not go into details for describing the homogenisation methods, which basically deal with averaged physical parameters, and serve well for static problems. But an important idea for describing waves in periodic structures must be stressed. The studies by Achenbach et al. [1968] and Sun et al. [1968] 
analysed dynamic behaviour of laminated composites by introducing the effective stiffness theory capable to describe dispersive effects. Later, a similar problem was studied by Ziegler [1977], who explained the mechanism of emerging stopping bands for harmonic waves as a result of combination of Floquet waves. Santosa and Symes [1991] derived a governing equation for waves in periodic composites which includes higher-order terms responsible for dispersion, like simple governing equations derived from lattice dynamics [Maugin 1999]. A similar problem for bilaminates is studied by Fish et al. [2002]. Nowadays, the homogenisation approach for such periodic composites is well developed; see for example [Craster et al. 2010].

Remark 2. In modelling of waves in microstructured solids, the inertial effects caused by the microstructure must be taken into account. This has been stressed already by Eringen [1964], who introduced the law of conservation of microinertia. This is characteristic to micromorphic models, strain gradient models, etc. In other words, kinetic energy must be attributed also to the microstructure, as in [Mindlin 1964; Mariano 2002; Engelbrecht et al. 2005].

\section{Models of waves in microstructured solids}

The diversity of models presented in the previous section means that " a unified continuum-mechanical description of materials with inherent microstructure is to date not available" [Kirchner and Steinmann 2004]. Nevertheless, there exists a guiding tool for the selection of a proper model: optical modes should be taken into account together with acoustic modes. As mentioned in [Chen et al. 2003],

The absence of optical branch is due to the neglecting of the atomic structure of crystal. From this viewpoint, classical continuum theory, the gradient theories, and the couple stress theories do not stem from the considerations of microstructure or micromotion and as a consequence, would break down if the micromotion and/or the microstructure become too significant to be neglected.

This is why we consider mainly approaches that include optical modes, at least in principle.

3.1. General frameworks. We start with most characteristic examples in the general setting.

3.1.1. Micromorphic solids. Mindlin [1964] formulated the linear micromorphic theory using two balance laws: one for macrostructure, another for microstructure. In the case of centrosymmetric, isotropic materials, these laws are 


$$
\begin{aligned}
\rho \dot{\boldsymbol{v}} & =\operatorname{div}(\boldsymbol{\sigma}+\boldsymbol{\tau})+\boldsymbol{f}, \\
\boldsymbol{I} \ddot{\boldsymbol{\psi}} & =\operatorname{div} \boldsymbol{\mu}+\boldsymbol{\tau}+\Phi,
\end{aligned}
$$

where $\rho$ is the density, $\boldsymbol{v}$ is the particle velocity, $\boldsymbol{I}$ is a microinertia tensor, $\boldsymbol{\psi}$ is the microdeformation tensor, $f$ is the body force, and $\Phi$ is the double force per unit volume. The corresponding stress tensors, namely, the Cauchy stress $\sigma$, the relative stress $\boldsymbol{\tau}$, and the double stress $\boldsymbol{\mu}$,

$$
\boldsymbol{\sigma} \equiv \frac{\partial W}{\partial \boldsymbol{\varepsilon}}, \quad \boldsymbol{\tau} \equiv \frac{\partial W}{\partial \boldsymbol{\gamma}}, \quad \boldsymbol{\mu} \equiv \frac{\partial W}{\partial \boldsymbol{\varkappa}},
$$

are defined, respectively, as derivatives of the free energy $W$ with respect to the classical strain tensor $\boldsymbol{\varepsilon}$, the relative deformation tensor $\boldsymbol{\gamma}$, and the microdeformation gradient $\mathcal{H}$ [Mindlin 1964],

$$
\boldsymbol{\varepsilon} \equiv \frac{1}{2}\left(\nabla \boldsymbol{u}+(\nabla \boldsymbol{u})^{T}\right), \quad \boldsymbol{\gamma} \equiv \nabla \boldsymbol{u}-\boldsymbol{\psi}, \quad \boldsymbol{x} \equiv \nabla \boldsymbol{\psi}
$$

In terms of the macrodisplacement $\boldsymbol{u}$, after choosing the quadratic free energy function $W$, Mindlin's model (1)-(2) results in

$$
\begin{aligned}
(\lambda+2 \mu)\left(1-l_{1}^{2} \nabla^{2}\right) \nabla \nabla \cdot \boldsymbol{u}-\mu\left(1-l_{2}^{2} \nabla^{2}\right) & \nabla \times \nabla \times \boldsymbol{u} \\
& =\rho\left(\ddot{\boldsymbol{u}}-h_{1}^{2} \nabla \nabla \cdot \ddot{\boldsymbol{u}}+h_{2}^{2} \nabla \times \nabla \times \ddot{\boldsymbol{u}}\right),
\end{aligned}
$$

where $l_{1}^{2}, l_{2}^{2}$ describe the elastic microstructural parameters and $h_{1}^{2}, h_{2}^{2}$ the microinertia, while $\lambda$ and $\mu$ are the Lamé parameters as in the classical theory of elasticity. This is known as Form I, in Mindlin's notation. Papargyri-Beskou et al. [2009] have followed [Mindlin 1964], and after certain simplifications get

$$
\left(1-g^{2} \nabla^{2}\right)\left[(\lambda+\mu) \nabla \nabla \cdot \boldsymbol{u}+\mu \nabla^{2} \boldsymbol{u}\right]=\rho\left(\ddot{\boldsymbol{u}}-h^{2} \nabla^{2} \ddot{\boldsymbol{u}}\right),
$$

where $g^{2}=l_{1}^{2}=l_{2}^{2}$ and $h^{2}=h_{2}^{1}=h_{2}^{2}$ govern the elastic microstructural and microinertia terms.

A more general model than that of [Mindlin 1964] is described in [Mariano 2002], with the effects of microstructure embedded into order parameters as

$$
\begin{aligned}
\operatorname{Div} \boldsymbol{T}+\boldsymbol{b}^{\mathrm{ni}} & =\rho \ddot{\boldsymbol{x}}, \\
\operatorname{Div} S-\boldsymbol{z}+\boldsymbol{\beta}^{\mathrm{ni}} & =\frac{d}{d t} \frac{\partial \chi}{\partial \dot{\varphi}}-\frac{\partial \chi}{\partial \varphi},
\end{aligned}
$$

where $\boldsymbol{x}$ is the placement field, $\varphi$ is the order parameter, $\boldsymbol{T}=\partial W / \partial \boldsymbol{F}$ is the first Piola-Kirchhoff stress tensor, $S=\partial W / \partial \nabla \varphi$ is the microstress tensor, $\boldsymbol{b}^{\text {ni }}$ is the noninertial bulk force, $\boldsymbol{z}=\partial W / \partial \varphi$ is the internal self-force, $\boldsymbol{\beta}^{\mathrm{ni}}$ is the noninertial external bulk interaction force, $\chi$ is the substructural kinetic coenergy density (the Legendre transform with respect to $\dot{\varphi}$ of the substructural kinetic energy density), and $\rho$ is the density of the macrostructure. The model is presented here in its original form [Mariano 2002], and all the notation can be found in the original paper. 
In the micromorphic theory of Mindlin, as well as in the multifield Mariano model, the balances of linear momentum for macroscale and microscale are introduced independently. This means that the introduced microdeformation or the corresponding order parameter play the role of an additional degree of freedom, causing obvious problems with boundary conditions.

3.1.2. Material formulation and internal variables. An alternative framework is provided by the internal variable approach. This approach is based on the canonical (or material) formulation of continuum mechanics. Following [Maugin 2006], we represent the canonical (material) momentum balance in the form

$$
\left.\frac{\partial \boldsymbol{P}}{\partial t}\right|_{X}-\operatorname{div}_{R} b=f^{\mathrm{int}}+\boldsymbol{f}^{\mathrm{ext}}+\boldsymbol{f}^{\mathrm{inh}},
$$

where $\boldsymbol{P}$ is material momentum, $\boldsymbol{b}$ the material Eshelby stress, and $\boldsymbol{f}^{\mathrm{int}}, \boldsymbol{f}^{\mathrm{ext}}$, and $f^{\text {inh }}$ are the material internal force, material external (body) force and the material inhomogeneity force, respectively. They are defined by

$$
\begin{gathered}
\boldsymbol{P}=-\rho_{0} \boldsymbol{v} \cdot \boldsymbol{F}, \quad \boldsymbol{b}=-\left(\mathscr{L}_{R}+\boldsymbol{T} \cdot \boldsymbol{F}\right), \quad \mathscr{L}=K-W, \\
\boldsymbol{f}^{\mathrm{inh}}=\left(\frac{1}{2} v^{2}\right) \nabla_{R} \rho_{0}-\left.\frac{\partial W}{\partial X}\right|_{\mathrm{expl}}, \\
\boldsymbol{f}^{\mathrm{ext}}=-\boldsymbol{f}_{0} \cdot \boldsymbol{F}, \quad \boldsymbol{f}^{\mathrm{int}}=\boldsymbol{T}:\left(\nabla_{R} \boldsymbol{F}\right)^{T}-\left.\nabla_{R} W\right|_{\mathrm{impl}},
\end{gathered}
$$

where $\boldsymbol{F}$ is the deformation gradient, $\rho_{0}$ is the matter density in the reference configuration, $\boldsymbol{v}$ is the velocity vector, $K$ is the kinetic energy density, $W$ is the free energy per unit reference volume, $\boldsymbol{T}$ is again the first Piola-Kirchhoff stress tensor, $f_{0}$ is the body force in the reference configuration. The subscript expl means taking the material gradient keeping the fields fixed (and thus extracting the explicit dependence on $\boldsymbol{X}$ ), while the subscript impl means taking the material gradient only through fields present in the function. The "dot" notation is used for the product of two tensors and colon denotes the tensor contraction. Equation (9) is called the pseudomomentum balance [Maugin 1993].

The canonical form of the energy conservation at any regular material point $\boldsymbol{X}$ in the body, for sufficiently smooth fields, has the form

$$
\left.\frac{\partial(S \theta)}{\partial t}\right|_{\boldsymbol{X}}+\nabla_{R} \cdot \boldsymbol{Q}=h^{\mathrm{int}}, \quad h^{\mathrm{int}}=\boldsymbol{T}: \dot{\boldsymbol{F}}-\left.\frac{\partial W}{\partial t}\right|_{\boldsymbol{X}},
$$

where $Q$ is the material heat flux, $S$ is the entropy density per unit reference volume, and $\theta$ is the absolute temperature. In addition, the Clausius-Duhem inequality is to be satisfied as

$$
-\left(\frac{\partial W}{\partial t}+S \frac{\partial \theta}{\partial t}\right)_{X}+\boldsymbol{T}: \dot{\boldsymbol{F}}+\nabla_{R}(\theta \boldsymbol{J})-\boldsymbol{S} \cdot \nabla_{R} \theta \geq 0
$$


where $\boldsymbol{S}$ is the entropy flux and $\boldsymbol{J}$ is the "extra entropy flux", which vanishes in most cases. Berezovski et al. [2011b] have used the dual internal variable concept [Ván et al. 2008] in addition to canonical equations (9) and (13). In this case, for example, the governing equation for the internal variable $\alpha$ (identified as the microdeformation tensor) is obtained by satisfying inequality (14) [Engelbrecht and Berezovski 2012]:

$$
\boldsymbol{I}_{m} \ddot{\boldsymbol{\alpha}}=\left(-\frac{\partial W}{\partial \boldsymbol{\alpha}}+\operatorname{div}_{R} \frac{\partial W}{\partial(\nabla \boldsymbol{\alpha})}\right),
$$

where $\boldsymbol{I}_{m}$ can be identified as the microinertia, calculated from geometry and state variables (for details, see [ibid.]).

In order to go further, the free energy function $W$ must be specified. The constraints for $W$ require positive definiteness for uniqueness and stability; it should be homogeneous and polyconvex in terms of the deformation gradient. In linear theory $W$ is a quadratic function; in the simplest version of nonlinear theory it is a cubic function. The regularity of energy densities is analysed in [Mariano 2002]. The specific question is how microdeformations (or the order parameter or internal variables) are described in the function $W$. This question must be answered with a full confidence about the internal structure but at the same time offers several opportunities for respective models (see below). For the description of deformation waves in microstructured solids, the inertia of the microstructure should be taken into account [Mindlin 1964; Mariano 2002].

Remark 3. Mindlin [1964] used Hamilton's principle for deriving the equations of motion combining total kinetic and potential energies and the work done by external forces. It is possible also to directly use the Euler-Lagrange formulation [Engelbrecht et al. 2005; Casasso and Pastrone 2010]. The two approaches yield the same equations of motion and the same pseudomomentum balance [Engelbrecht et al. 2006].

3.2. One-dimensional models. The classical one-dimensional wave equation possesses the well-known d'Alembert solution. It is of considerable interest to use the one-dimensional setting also for describing microstructured solids in order to understand possible effects in the most transparent way. Certainly, one notes the price of simplifications compared with three-dimensional models: the oversimplified description of rotational effects, as in the Cosserats' model, the emerging artefact between scalar and covectorial character of energy equation and the balance of momentum [Maugin 2011a] and simplification of geometry of solids. On the other hand, the appearance of new terms in the wave equation can cast more light on dispersive, nonlinear, and other effects. A typical example in this sense is the Boussinesq-type equation [Christov et al. 2007; Engelbrecht et al. 2011]. 
3.2.1. Structure of equations. The models briefly described in Section 3.1 result in systems of partial differential equations. In the one-dimensional case with a single microstructure, this means that one obtains a system of two equations. If we now take displacement $u=u_{1}$ and the microdeformation $\varphi=\varphi_{11}$ according to the Mindlin model [1964], then the kinetic energy density $K$ and the potential energy density $W$ are

$$
K=\frac{1}{2} \rho u_{t}^{2}+\frac{1}{2} I \varphi_{t}^{2}, \quad W=W\left(u_{x}, \varphi, \varphi_{x}\right),
$$

where $\rho$ and $I$ denote macroscopic density and microinertia, respectively, and indices denote derivatives. The corresponding Euler-Lagrange equations have the general form

$$
\begin{aligned}
& \left(\frac{\partial \mathscr{L}}{\partial u_{t}}\right)_{t}+\left(\frac{\partial \mathscr{L}}{\partial u_{x}}\right)_{x}-\frac{\partial \mathscr{L}}{\partial u}=0, \\
& \left(\frac{\partial \mathscr{L}}{\partial \varphi_{t}}\right)_{t}+\left(\frac{\partial \mathscr{L}}{\partial \varphi_{x}}\right)_{x}-\frac{\partial \mathscr{L}}{\partial \varphi}=0,
\end{aligned}
$$

where the Lagrangian density is given by $\mathscr{L}=K-W$. The simplest potential energy function is a quadratic function

$$
W=\frac{1}{2}(\lambda+2 \mu) u_{x}^{2}+A \varphi u_{x}+\frac{1}{2} B \varphi^{2}+\frac{1}{2} C \varphi_{x}^{2},
$$

where $\lambda, \mu$ are Lamé parameters (in order to keep notations from classical elasticity) and $A, B, C$ are other material constants.

The governing equations are now [Engelbrecht et al. 2005]

$$
\begin{aligned}
\rho u_{t t} & =(\lambda+2 \mu) u_{x x}+A \varphi_{x}, \\
I \varphi_{t t} & =C \varphi_{x x}-A u_{x}-B \varphi,
\end{aligned}
$$

which is the simplest Mindlin-type (micromorphic) model. A similar model is used in [Porubov et al. 2009; Casasso and Pastrone 2010]. Huang and Sun [2008] have derived the governing equations for waves along the layers of a bimaterial layered medium. In terms of the displacement $U$ and "kinematic variable" $\Phi$, the governing system of equations in a micromorphic case is

$$
\begin{aligned}
& k_{1} U_{t t}=k_{2} U_{x x}+k_{2} \Phi_{x}, \\
& k_{3} \Phi_{t t}=k_{4} \Phi_{x x}-k_{2} U_{x}-k_{2} \Phi,
\end{aligned}
$$

where $k_{1}, k_{2}, k_{3}, k_{4}$ reflect elastic and geometric characteristics of layers. The system of equations (20)-(21) is very similar to the system (22)-(23).

Maugin [1999] has derived a model of a chain of dumbbells (see also [Askar 1986]) which exhibits transverse displacements $U$ and rotations $\psi$. After continualisation, the governing equations are 


$$
\begin{aligned}
\rho_{0} U_{t t} & =(\mu+\chi) U_{x x}+\chi \psi_{x}, \\
j \psi_{x x} & =\alpha \psi_{x x}-\chi U_{x}-\chi \psi,
\end{aligned}
$$

where coefficients are related to the mass $m$, chain scale $a$ and stiffness $k$. Note that here $j$ is the microinertia density and $\chi$ and $\alpha$ are micropolar coefficients.

Again, the structure of the equations is the same as above. In order to compare these (and other) results within one framework, we shall focus now on models in the form of single equation, derived directly from three-dimensional models (5) or (6), or from systems of equations like (20)-(21) or (22)-(23). Let us introduce a wave operator

$$
\mathscr{L}_{w}=u_{t t}-c_{i}^{2} u_{x x},
$$

where $c_{i}$ is a velocity, and the function

$$
\mathscr{F}_{w}=\mathscr{F}_{w}\left(u_{x x}, u_{x x x x}, u_{x x t t}, \ldots\right),
$$

leaving the coefficients of derivative aside. Then the models derived for describing the one-dimensional wave propagation in microstructured solids can be summarised by either

$$
\mathscr{L}_{w}=\mathscr{F}_{w}(\cdot)
$$

or, more explicitly,

$$
\mathscr{L}_{w}=\left(\mathscr{L}_{j w}\right)_{x x}+\left(\mathscr{L}_{k w}\right)_{t t}+\mathscr{F}_{w}(\cdot),
$$

where $\mathscr{L}_{j w}, \mathscr{L}_{k w}$ have velocities $c_{j}, c_{k}$, respectively (see Whitham [1974]). The presentation (29) can be called hierarchical since it involves several wave operators, and in general $\mathscr{L}_{i w}=\mathcal{O}(\epsilon)$, where $\epsilon$ is a small parameter. In general terms, $\mathscr{F}_{w}$ is also of accuracy $\mathcal{O}(\epsilon)$.

3.2.2. Classification of models. Models of dispersive waves in terms of $\mathscr{L}_{w}$ and $\mathscr{F}_{w}$ are collected in Table 1, and the corresponding models in terms of $\mathscr{L}_{w}$ and $\mathscr{L}_{j w}$ are presented in Table 2.

The models listed in Tables 1 and 2 are derived using various assumptions and procedures, but they all include higher-order derivatives in space and/or in time, or mixed space-time ones. All these derivatives are even, which shows that the models are conservative and that they display dispersive effects (see Section 5). These effects are reflected first in phase and group velocities, and second in wave profiles.

Table 2 demonstrates the structures of models using the wave operators (26). The basic idea behind introducing such operators is the possibility to stress the importance of scaling [Engelbrecht et al. 2006]. Namely, in this case the wave hierarchies can be formulated [Whitham 1974]. The general structure of models is then

$$
\mathscr{L}_{1 w}=\delta\left(\mathscr{L}_{1 w}\right)_{x x}+\delta^{2}\left(\mathscr{L}_{2 w}\right)_{x x x x}+\cdots,
$$




\begin{tabular}{|l|l|}
\hline $\mathscr{F}_{w}(\cdot)$ & $\begin{array}{l}\text { Sources } \\
0\end{array}$ \\
$u_{x x x x}$ & $\begin{array}{l}\text { Classical wave equation } \\
\text { [Santosa and Symes 1991], [Maugin 1995], } \\
\text { [Erofeyev 2003], [Andrianov et al. 2010], } \\
\text { [dell'Isola et al. 2012], [Andrianov et al. 2013] } \\
\text { [Pichugin et al. 2008], [Andrianov et al. 2011] } \\
\text { [Love 1944], [Maugin 1995], [Wang and Sun 2002] } \\
u_{x x x x}, u_{6 x} \\
u_{x x t t} \\
u_{x x x x}, u_{x x t t}\end{array}$ \\
$\begin{array}{l}\text { [Askes and Metrikine 2002], [Pastrone et al. 2004], } \\
\text { [Metrikine 2006], [Papargyri-Beskou et al. 2009], } \\
\text { [Porubov et al. 2009], [Challamel et al. 2009], } \\
\text { [Polyzos and Fotiadis 2012] } \\
\text { [Engelbrecht and Pastrone 2003], } \\
\text { [Metrikine 2006], [Polyzos and Fotiadis 2012], } \\
\text { [Pichugin et al. 2008] } \\
u_{x x x x}, u_{x x t t}, u_{x x} \\
u_{x x x x}, u_{x x t t}, u_{t t t t} \\
u_{x x t t}, u_{t t t t} \\
u_{x x x x}, u_{x x t t}, u_{6 x}\end{array}$ \\
\hline
\end{tabular}

Table 1. Models of dispersive waves in the form $\mathscr{L}_{w}=\mathscr{F}_{w}, \mathscr{L}_{w}=u_{t t}-c_{0}^{2} u_{x x}$.

\begin{tabular}{|l|l|l|l|}
\hline$\left(\mathscr{L}_{j w}\right)$ & $\left(\mathscr{L}_{k w}\right)$ & $\mathscr{F}_{w}$ & Source \\
$\left(u_{t t}-c_{j}^{2} u_{x x}\right)_{x x}$ & & $u_{x x}$ & [Engelbrecht et al. 2005] \\
& & & [Engelbrecht and Salupere 2014] \\
& $\left(u_{t t}-c_{i}^{2} u_{x x}\right)_{t t}$ & & [Maugin 1995] (here $\left.c_{k}=c_{i}\right)$ \\
$\left(u_{t t}-c_{i}^{2} u_{x x}\right)_{x x}$ & $\left(u_{t t}-c_{i}^{2} u_{x x}\right)_{t t}$ & $u_{x x}$ & [Engelbrecht et al. 2005] \\
& & & [Berezovski et al. 2011b] \\
$\left(u_{t t}-c_{i}^{2} u_{x x}\right)_{x x}$ & & $u_{x x x x}$ & [Berezovski et al. 2011b] \\
$\left(u_{t t}-c_{i}^{2} u_{x x}\right)_{x x}$ & $\left(u_{t t}-c_{i}^{2} u_{x x}\right)_{t t}$ & $u_{x x}, u_{x x x x}$ & [Berezovski et al. 2011b] \\
$\left(u_{t t}-c_{i}^{2} u_{x x}\right)_{x x}$ & $\left(u_{t t}-c_{i}^{2} u_{x x}\right)_{t t}$ & $u_{x x x x}$ & [Engelbrecht et al. 2005] \\
\hline
\end{tabular}

Table 2. Models of dispersive waves in the form $\mathscr{L}_{w}=\left(\mathscr{L}_{j w}\right)_{x x}+\left(\mathscr{L}_{k w}\right)_{t t}+\mathscr{F}_{w}$.

where $\delta$ is related to the ratio $L_{0} / l$. Such a hierarchy explicitly demonstrates the dependence of the macrobehaviour on microstructure. If $\delta$ is small then waves "feel" more the properties of the macrostructure, and the influence of the microstructure is of a perturbative character. If, however, $\delta$ is large, then waves "feel" more the properties of the microstructure. The wave hierarchies are analysed in several 
studies [Whitham 1974; Engelbrecht et al. 2006; Casasso and Pastrone 2010] and an overview presented by Engelbrecht and Salupere [2014].

The most complicated case - model 5 of Table 2 - has been derived by Berezovski et al. [2011a] by using the concept of dual internal variables $\varphi_{1}$ and $\varphi_{2}$ [Ván et al. 2008]. Here, $\varphi_{1}$ is identified as a microdeformation and $\varphi_{2}$ as its rate. The energy function has the quadratic form

$$
W=\frac{1}{2} \rho c^{2} u_{x}^{2}+A u_{x} \varphi_{1}+A^{\prime} u_{x} \varphi_{2}+\frac{1}{2} B \varphi_{1}^{2}+\frac{1}{2} C\left(\varphi_{1}^{2}\right)_{x}+\frac{1}{2} D \varphi_{2}^{2},
$$

where $\rho$ is the density and $c$ is the velocity in the macrostructure; the coefficients $A$ and $A^{\prime}$ characterise coupling, and $B, C, D$ the microstructure. The corresponding dispersive wave equation has the form

$$
u_{t t}-c^{2} u_{x x}=\frac{C}{B}\left(u_{t t}-c^{2} u_{x x}\right)_{x x}-\frac{I}{B}\left(u_{t t}-c^{2} u_{x x}\right)_{t t}+\frac{A^{\prime 2}}{\rho B} u_{x x x x}-\frac{A^{2}}{\rho B} u_{x x} .
$$

The right-hand side of (32) includes the space and time derivatives of the wave operator $\mathscr{L}_{w}=u_{t t}-c^{2} u_{x x}$ and additional terms with coupling coefficients. Note that (32) can be rewritten in terms of different wave operators as

$$
u_{t t}-\left(c^{2}-c_{A}^{2}\right) u_{x x}=\frac{C}{B}\left(u_{t t}-\left(c^{2}-c_{c}^{2}\right) u_{x x}\right)_{x x}-\frac{I}{B}\left(u_{x x}-c^{2} u_{x x}\right)_{t t},
$$

where $c_{A}^{2}=A^{2} / \rho B, c_{c}^{2}=A^{\prime 2} / \rho C$. If $A^{\prime}=0$ then (32) yields, in a shorter form obtained by asymptotic analysis [Engelbrecht et al. 2005],

$$
u_{t t}-\left(c^{2}-c_{A}^{2}\right) u_{x x}=p^{2} c_{A}^{2}\left(u_{t t}-c_{1}^{2} u_{x x}\right)_{x x},
$$

where $c_{1}^{2}=C / I B, p^{2}=I / B$.

Remark 4. The models in Section 3.2 are one-dimensional with a clear threedimensional background. Similar models are known for rods [Samsonov 2001; Porubov 2003], where the higher-order derivatives in governing equations in terms of the displacement appear due to geometrical considerations (effects of the transverse displacement). As far as dispersive effects are of a different character, the governing equation is called the "double" dispersion equation [Samsonov 2001]. The combination of microstructural effects and geometrical characteristics of rods is studied in [Porubov 2000].

\section{Generalisation of models}

The mathematical models described in Section 3 involved one microstructure beside the macrostructure; this microstructure is homogeneously distributed over the space, the dissipative effects are not accounted for, and the governing equations are linear. Clearly, these assumptions need more attention and, if needed, to be 
changed. In what follows, possible generalisations are described. Dissipative models will not be considered here; attention is paid to thermoelasticity, i.e., to coupled fields with energy transfer.

4.1. Multiscale models. In reality, there are cases when a microstructure includes another microstructure at a smaller scale [Engelbrecht et al. 2006], or there are two microstructures in parallel with different properties [Berezovski et al. 2010]. The first case (a scale within a scale) may be called hierarchical microstructures and the second case concurrent microstructures.

For a hierarchical microstructure, the free energy function is taken in the form [Engelbrecht et al. 2006; Berezovski et al. 2010]

$$
\begin{aligned}
W=\frac{1}{2}(\lambda+2 \mu) u_{x}^{2}+A_{1} \varphi_{1} u_{x}+\frac{1}{2} B_{1} \varphi_{1}^{2} & +\frac{1}{2} C_{1}\left(\varphi_{1}\right)_{x}^{2} \\
& +\frac{1}{2} A_{12}\left(\varphi_{1}\right)_{x} \varphi_{2}+\frac{1}{2} B_{2} \varphi_{2}^{2}+\frac{1}{2} C_{2}\left(\varphi_{2}\right)_{x}^{2},
\end{aligned}
$$

where $\varphi_{1}$ and $\varphi_{2}$ are microdeformations ( $\varphi_{2}$ within $\left.\varphi_{1}\right)$ and $A_{1}, B_{1}, B_{2}, C_{1}, C_{2}, A_{12}$ are coefficients. Then the governing equations are (see also [Casasso and Pastrone 2010])

$$
\begin{aligned}
\rho u_{t t} & =(\lambda+2 \mu) u_{x x}+A_{1}\left(\varphi_{1}\right)_{x}, \\
I_{1}\left(\varphi_{1}\right)_{t t} & =C_{1}\left(\varphi_{1}\right)_{x x}-A_{1} u_{x}-B_{1} \varphi_{1}+A_{12}\left(\varphi_{2}\right)_{x}, \\
I_{2}\left(\varphi_{2}\right)_{t t} & =C_{2}\left(\varphi_{2}\right)_{x x}-A_{12}\left(\varphi_{1}\right)_{x}-B_{2} \varphi_{2},
\end{aligned}
$$

where $I_{1}$ and $I_{2}$ are the corresponding microinertias.

For a concurrent microstructure, the free energy is [Berezovski et al. 2010]

$$
\begin{aligned}
W=\frac{1}{2}(\lambda+2 \mu) u_{x}^{2}+A_{1} \varphi_{1} u_{x} & +\frac{1}{2} B_{1} \varphi_{1}^{2}+\frac{1}{2} C_{1}\left(\varphi_{1}\right)_{x}^{2} \\
& +A_{12}\left(\varphi_{1}\right)_{x} \varphi_{2}+\frac{1}{2} B_{2} \varphi_{2}^{2}+\frac{1}{2} C_{2}\left(\varphi_{2}\right)_{x}^{2}+A_{2} \varphi_{2} u_{x},
\end{aligned}
$$

where now $\varphi_{1}$ and $\varphi_{2}$ are parallel microstructure (the same scale). The governing equations become

$$
\begin{aligned}
\rho u_{t t} & =(\lambda+2 \mu) u_{x x}+A_{1}\left(\varphi_{1}\right)_{x}+A_{2}\left(\varphi_{2}\right)_{x}, \\
I_{1}\left(\varphi_{1}\right)_{t t} & =C_{1}\left(\varphi_{1}\right)_{x x}+A_{12}\left(\varphi_{2}\right)_{x}-A_{1} u_{x}-B_{1} \varphi_{1}, \\
I_{2}\left(\varphi_{2}\right)_{t t} & =C_{2}\left(\varphi_{2}\right)_{x x}-A_{12}\left(\varphi_{1}\right)_{x}-A_{2} u_{x}-B_{2} \varphi_{2} .
\end{aligned}
$$

If $A_{12} \neq 0$ then the microstructures are coupled; if, however, $A_{12}=0$, then both microstructures are coupled with the macrostructure but not coupled with each other [Berezovski et al. 2010]. In the latter case, after reformulating the system of equations (36)-(38) in the form of a single equation and using asymptotic analysis, the analogue to (33) can be obtained as [Engelbrecht et al. 2006] 


$$
\begin{aligned}
& u_{t t}-\left(c_{0}^{2}-a_{A 1}^{2}\right) u_{x x} \\
& \quad=p_{1}^{2} c_{A 1}^{2}\left[u_{t t}-\left(c_{1}^{2}-c_{A 2}^{2}\right) u_{x x}\right]_{x x}-p_{1}^{2} c_{A 1}^{2} c_{A 2}^{2}\left(u_{t t}-c_{2}^{2} u_{x x}\right)_{x x x x},
\end{aligned}
$$

where $c_{A 1}^{2}=A_{1}^{2} / \rho B_{1}, c_{A 2}^{2}=A_{2}^{2} / \rho B_{2}, c_{1}^{2}=C_{1} / I_{1}, p_{1}^{2}=I_{1} / B_{1}, p_{2}^{2}=I_{2} / B_{2}$. One should note the appearance of the sixth-order derivatives in (43), as in the model derived from lattice dynamics in the continuum limit [Maugin 1999]. However, here $u_{\text {ttxxxx }}$ is also involved because of the inertia of the second microstructure.

There are many studies concerning multiscales in terms of building of atomisticcontinuum models (see, for example, [Liu et al. 2010] and the references therein). Such an approach is needed because, in design of structural elements, some areas like crack tips, plastic zones, thin layers, etc., need smaller spatial scales compared with the whole. That is why mixed atomistic-continuum models are derived. Here, however, multiscale is understood as excitation-dependent (i.e., frequencydependent). The governing parameter is $L_{0} / l$, which gives the weight to different terms in models, and consequently is the reason for the appearance of different physical effects.

4.2. Nonlinear models. Contemporary technology is often characterised by intensive and high-speed impacts. That is why nonlinearities should be accounted for in mathematical models. This means that the full deformation tensor involves nonlinear terms and the stress-strain should also be nonlinear [Eringen 1962]. As shown by Engelbrecht [1997], the physical nonlinearities (stress-strain relations) for most materials are stronger than geometrical (deformation tensor), and, therefore, we limit ourselves here to physical nonlinearities only. This is easily formulated in terms of the free energy function $W$. Note, however, that we deal with onedimensional problems, and for three-dimensional problems such an assumption needs careful analysis. Following the model (20)-(21), instead of the free energy function (18), we can postulate [Engelbrecht et al. 2005]

$$
W=\frac{1}{2}(\lambda+2 \mu) u_{x}^{2}+A \varphi u_{x}+\frac{1}{2} B \varphi^{2}+\frac{1}{2} C \varphi_{x}^{2}+\frac{1}{6} N u_{x}^{3}+\frac{1}{6} M \varphi_{x}^{3},
$$

where now $N$ and $M$ are nonlinear parameters for macro- and microstructure, respectively. In this case, the governing equations are

$$
\begin{gathered}
\rho u_{t t}=(\lambda+2 \mu) u_{x x}+N u_{x} u_{x x}+A \varphi_{x}, \\
I \varphi_{t t}=C \varphi_{x x}+M \varphi_{x} \varphi_{x x}-A u_{x}-B \varphi .
\end{gathered}
$$

Porubov et al. [2009] have derived the same model, linking $N$ and $M$ to Murnaghan's moduli. From (45) and (46) we get

$$
u_{t t}-\left(c^{2}-c_{A}^{2}\right) u_{x x}-k_{1}\left(u_{x}^{2}\right)_{x}=p^{2} c_{A}^{2}\left(u_{t t}-c_{1}^{2} u_{x x}\right)_{x x}+k_{2}\left(u_{x x}^{2}\right)_{x x},
$$

where $k_{1}$ and $k_{2}$ are coefficients (compare with (33)). Equation (47) in terms of 
the deformation $v=u_{x}$ reads

$$
v_{t t}-\left(c^{2}-c_{A}^{2}\right) v_{x x}-k_{1}\left(v^{2}\right)_{x x}=p^{2} c_{A}^{2}\left(v_{t t}-c_{1}^{2} v_{x x}\right)_{x x}+k_{2}\left(v_{x}^{2}\right)_{x x x} .
$$

This equation will be analysed later.

Several modifications of (47) are derived under various assumptions. Maugin [1999] has described the continualisation of discrete lattices and obtained

$$
u_{t t}-c^{2} u_{x x}+c^{2} \beta a u_{x} u_{x x}-\frac{c^{2} a^{2}}{12} u_{x x x x}=0,
$$

where $a$ is lattice spacing and $\beta$ is related to the potential. Andrianov et al. [2013] have derived a similar model for a layered composite and Erofeyev [2003] for a medium with coupled stresses. In these models, nonlinear terms stem from the macrostructure. It is certainly possible to assume that nonlinearity is essential only at the level of the microstructure. Then the corresponding governing equation reads [Engelbrecht and Pastrone 2003]

$$
u_{t t}-\left(c^{2}-c_{A}^{2}\right) u_{x x}=p^{2} c_{A}^{2}\left(u_{t t}-c_{1}^{2} u_{x x}\right)_{x x}+k_{2}\left(u_{x x}^{2}\right)_{x x} .
$$

All models (47)-(50) are of the Boussinesq-type. Christov et al. [1996; 2007] have stated that the Boussinesq paradigm grasps the following effects: (a) bidirectionality of waves; (b) nonlinearity (of any order); (c) dispersion (of any order, modelled by space and time derivatives of the fourth order at least). The recent results of studies are summarised by Christov et al. [2007], and with a special attention to microstructured solids by Engelbrecht et al. [2011].

Remark 5. The nonlinear governing equation for rods derived by Samsonov [2001] and Porubov [2003] belong also to the family of Boussinesq equations. In this case the nonlinearity is quadratic, like the nonlinearity of the macrostructure (equations (47) and (48)) in microstructured materials, but it may also be cubic [Porubov and Maugin 2005].

4.3. Thermoelastic models. The classical thermoelastic theory combines the elastic behaviour of homogeneous media with heat conduction, which is usually governed by Fourier's law (see, for example, [Nowacki 1972]). The difference between elastic deformation of a solid and heat conduction consists in that the former is a conservative process without dissipation, whereas the latter is a dissipative one. Bearing in mind microstructured materials, it is possible to use the concept of internal variables to construct mathematical models for governing the wave motion. This is achieved by the dual internal variable theory [Ván et al. 2008], which permits modelling of the dissipation effects due to the microstructure in thermoelastic solids [Berezovski et al. 2011b; Engelbrecht and Berezovski 2012]. The dissipation is associated with microtemperature, i.e., fluctuations of temperature due to the 
difference of thermal characteristics of the macro- and microstructure in a solid. Here we present two models: the first one dealing with microtemperature only, and the second one with microdeformation and microtemperature simultaneously.

First, following [Berezovski et al. 2011b] and [Berezovski and Engelbrecht 2013], we postulate the free energy function $W$ as

$$
W=\frac{1}{2}(\lambda+2 \mu) u_{x}^{2}-\frac{\rho c_{p}}{2 \theta_{0}}\left(\theta-\theta_{0}\right)^{2}+m\left(\theta-\theta_{0}\right) u_{x}+Q \varphi_{x} u_{x}+\frac{1}{2} P \varphi_{x}^{2}+\frac{1}{2} D \psi^{2},
$$

where $c_{p}$ is the heat capacity, $m=-\alpha(3 \lambda+2 \mu), \alpha$ is the dilatation coefficient, $\theta$ is the temperature, $\theta_{0}$ is the reference temperature. The first internal variable $\varphi$ is the microtemperature and the second internal variable $\psi$ is the rate of the first one; $Q, P, D_{2}$ are, as before, the material parameters. Leaving aside the details (see references above), the governing equations are

$$
\begin{aligned}
\rho u_{t t} & =(\lambda+2 \mu) u_{x x}+m \theta_{x}+Q \varphi_{x x}, \\
L \varphi_{t t}+R \varphi_{t} & =P \varphi_{x x}+Q u_{x x}, \\
\rho c_{p} \theta_{t}-\left(k \theta_{x}\right)_{x} & =m \theta_{0} u_{x t}+R \varphi_{t}^{2},
\end{aligned}
$$

where $k$ is the thermal conductivity and $R, L$ are the material parameters related to conductance, which are obtained from satisfying the dissipation inequality. Note that the heat conduction is governed by the parabolic equation (54), while the microtemperature is governed by the hyperbolic equation (53).

Second, following Berezovski et al. [2011b], we use the dual internal variables; the first pair, $\varphi_{1}$ and $\varphi_{2}$, are related to microdeformation, and the second pair, $\psi_{1}$ and $\psi_{2}$, to microstructure. Then the free energy function $W$ is

$$
\begin{aligned}
W=\frac{1}{2}(\lambda+2 \mu) u_{x}^{2} & -\frac{\rho c_{p}}{2 \theta_{0}}\left(\theta-\theta_{0}\right)^{2}+m\left(\theta-\theta_{0}\right) u_{x}+A \varphi_{1} u_{x}+\frac{1}{2} B \varphi_{1}^{2} \\
& +\frac{1}{2} C\left(\varphi_{1}^{2}\right)_{x}+\frac{1}{2} D_{1} \varphi_{2}^{2}+Q\left(\psi_{1}\right)_{x} u_{x}+\frac{1}{2} P\left(\psi_{1}^{2}\right)_{x}+\frac{1}{2} D_{2} \psi_{2}^{2},
\end{aligned}
$$

where $A, B, C, D_{1}, Q, P, D_{2}$ are, as before, the material parameters. Now the governing equations are

$$
\begin{aligned}
\rho u_{t t} & =(\lambda+2 \mu) u_{x x}+m \theta_{x}+A\left(\varphi_{1}\right)_{x}+Q\left(\varphi_{2}\right)_{x x}, \\
I\left(\varphi_{1}\right)_{t t} & =C\left(\varphi_{1}\right)_{x x}-A u_{x}-B \varphi_{1}, \\
L\left(\varphi_{2}\right)_{t t}+R\left(\varphi_{2}\right)_{t} & =P\left(\varphi_{2}\right)_{x x}+Q u_{x x}, \\
\rho c_{p} \theta_{t}-\left(k \theta_{x}\right)_{x} & =m \theta_{0} u_{x t}+R\left(\varphi_{2}^{2}\right)_{t} .
\end{aligned}
$$

For details of the derivation, see [Berezovski et al. 2014]. This model contains two hyperbolic equations for microeffects: Equation (57), for microinertia, and Equation (58), for microtemperature. These equations are not coupled, but both of them are coupled with the balance of momentum (56). The fourth equation, (59), 
which models the heat conduction at the macrolevel, is parabolic and related only to the microtemperature, while microdeformation is nondissipative.

4.4. Models for inhomogeneous microstructure. Previous models were derived using the assumption of the homogeneity of the microstructure. In many practical application, this assumption must be refined. This is the case, for example, of functionally graded materials (FGMs) which are made up of two or more materials (constituent phases) combined in solid states [Birman and Byrd 2007; Mahamood et al. 2012]. The specimens made of FGMs can have a thin coating of a parent material or a special distribution within a bulk material [Yin et al. 2004]. Such inhomogeneities should be taken into account also in deriving the governing equations.

The simplest way to do that is to modify the well-known Mindlin model. In this case, starting from the conventional free energy function

$$
W=\frac{1}{2}(\lambda+2 \mu) u_{x}^{2}+A \varphi u_{x}+\frac{1}{2} B \varphi^{2}+\frac{1}{2} C \varphi_{x}^{2},
$$

we assume that $A=A(x), B=B(x)$, and $C=C(x)$. Then the governing equations yield (compare with equations (20) and (21))

$$
\begin{aligned}
\rho u_{t t} & =(\lambda+2 \mu) u_{x x}+A \varphi_{x}+A_{x} \varphi, \\
I \varphi_{t t} & =C \varphi_{x x}-A u_{x}-B \varphi+C_{x} \varphi_{x} .
\end{aligned}
$$

Here, as before, $\varphi$ denotes the microdeformation, the elements of the microstructure are of the same size $(I=$ const $)$, but the variation of the microstructure is emphasised by $A_{x}$ and $C_{x}$.

\section{Wave dispersion}

The main aim of applying various theories to derive mathematical models is to get closer to reality. It has been shown in Sections 3 and 4 that inertia of microstructure(s) leads to higher-order time derivatives, the elasticity of microstructure(s) to higher-order space derivatives, and the coupling of macro- and microstructures results in the changes of velocities. The latter phenomenon is demonstrated also by numerical simulation of waves in metal-ceramic composites by using the finite volume method [Engelbrecht et al. 2005]. In this case, the physical parameters were assigned to every volume element in a material. The changes in the volume fraction $f=V_{c} / V$ are directly reflected in changes of velocities, where $V_{c}$ is the volume of ceramic particles and $V$ is the total volume.

In what follows, we explain how introduced microstructure models affect the macrobehaviour of waves: dispersion, wave profiles, frequencies, velocities, spectra, etc. As we have seen, the classical nondispersive wave equation is modified for processes in microstructured solids. The included even-order higher derivatives (fourth-order, sixth-order, etc.) lead to dispersion of waves. In discrete systems 
the dispersion analysis is carried out by Brillouin [1946] and Askar [1986]. For diatomic chains they noticed the existence of acoustical and optical branches of the dispersion relation. Mindlin [1964] and Eringen [1972] have described the behaviour of the dispersion curves in microstructured materials, including also acoustical and optical branches. There are many studies to be noted [Huang and Sun 2008; Metrikine 2006; Andrianov et al. 2013] in dispersion analysis, but the most detailed studies are presented by Papargyri-Beskou et al. [2009] and Berezovski et al. [2013].

These studies demonstrate that the typical dispersion curves are convex. However, given the structural characteristics of solids, the periodic character of dispersion curves, as shown by Brillouin [1946] for a discrete chain, is not observed except in some limit cases [Pichugin et al. 2008]. In most models where the assumptions about length scales involve long waves (small wave numbers), the dispersion curves in a short-wave limit tend to some asymptotes corresponding to certain velocities [Engelbrecht et al. 2005; Metrikine and Askes 2002; Berezovski et al. 2011a]. This is explicitly seen from the analysis of group and phase velocities [Papargyri-Beskou et al. 2009; Berezovski et al. 2013]. If short waves are close to nanoscale in the length, then phonon-like dispersion curves are closer to the results of Brillouin [Maranganti and Sharma 2007].

5.1. Dispersion relations. Papargyri-Beskou et al. [2009] derived the following dispersion relation, following [Mindlin 1964] (see (6) in the one-dimensional setting):

$$
\omega^{2}=c^{2} k^{2}\left(1+g^{2} k^{2}\right)\left(1+h^{2} k^{2}\right)^{-1}, \quad c^{2}=(\lambda+2 \mu) / \rho,
$$

where $\omega, k$ are the frequency and the wave number, respectively, and $g^{2}, h^{2}$ are the microstructural elasticity and microinertia coefficients. The corresponding dispersion curves are shown in Figure 1. It is concluded that the dispersion is physically acceptable only if $h^{2}>g^{2}$. This conclusion stresses the importance of the microinertia. The main deficiency of the strain-gradient model is the absence of the optical branch of the dispersion curve.

A more realistic dispersion relation follows from (33), with $A^{\prime}=0$ for the sake of simplicity (see [Berezovski et al. 2013]):

$$
\omega^{2}=\left(c^{2}-c_{A}^{2}\right) k^{2}+p^{2}\left(\omega^{2}-c^{2} k^{2}\right)\left(\omega^{2}-c_{1}^{2} k^{2}\right) .
$$

The corresponding dispersion curves are shown in Figure 2 [Berezovski et al. 2013]. In this figure, the dispersion curve computed from the relation

$$
\omega^{2}=\left(c^{2}-c_{A}^{2}\right) k^{2}-p^{2} c_{A}^{2}\left(w^{2}-c_{1}^{2} k^{2}\right)^{2}
$$




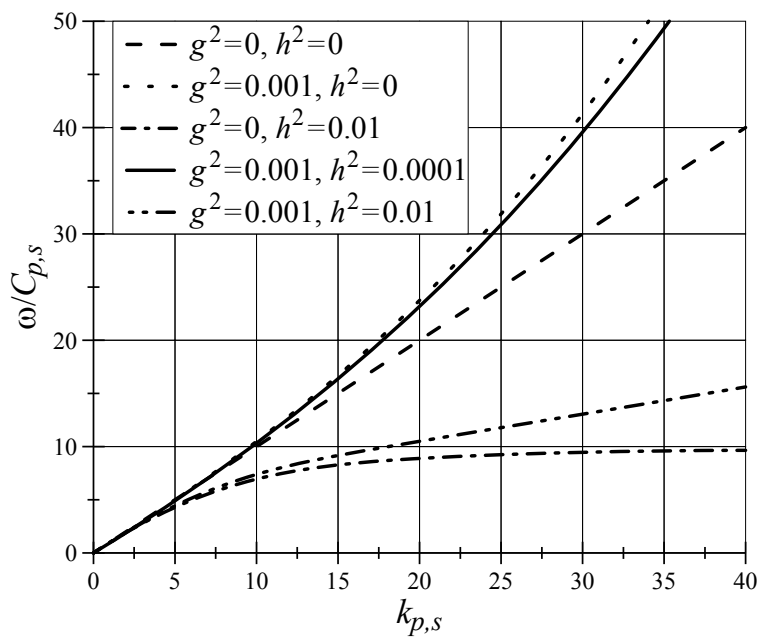

Figure 1. Dispersion curves of the $\omega / C_{p, s}$ versus wavenumber $k_{p, s}$ type for elastic medium with microstructure obeying Equation (63). Adapted from [Papargyri-Beskou et al. 2009].

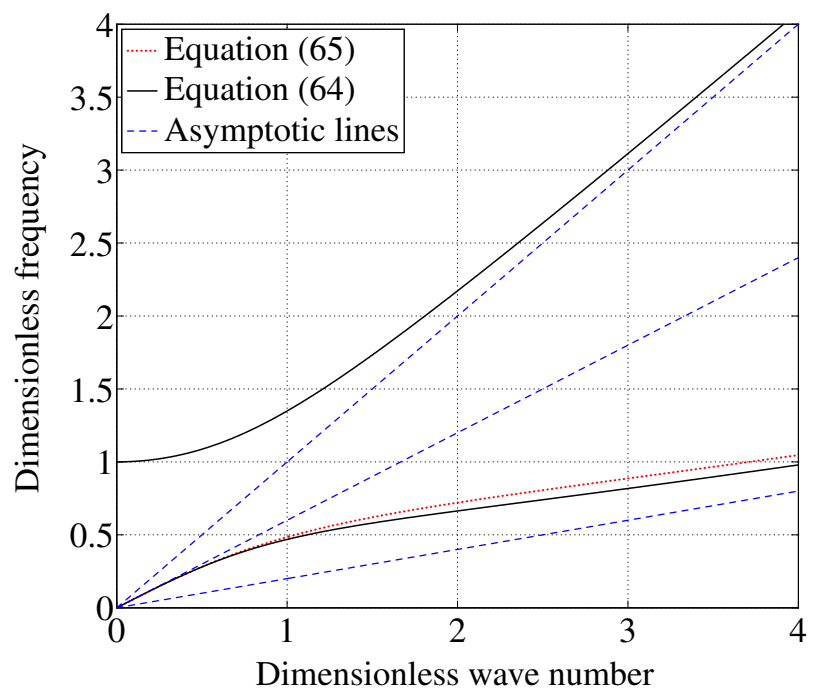

Figure 2. Dispersion curves in case of $c_{\mathrm{gr}}<c_{\mathrm{ph}}\left(c_{A}=0.8 c, c_{1}=0.2 c\right)$.

following from (34) is also shown. The asymptotes in Figure 2 reflect the dimensionless velocities: $\omega=k, \omega=c_{1} k / c, \omega=c_{R} k / c$, where $c_{1}^{2}=C / I, c_{R}^{2}=c^{2}-c_{A}^{2}$. These velocities play a role in the case of shorter waves.

Dispersion relation (64), which reflects the behaviour of waves modelled by (33), leads to two dispersion curves - one branch is acoustic (lower branch), another one 


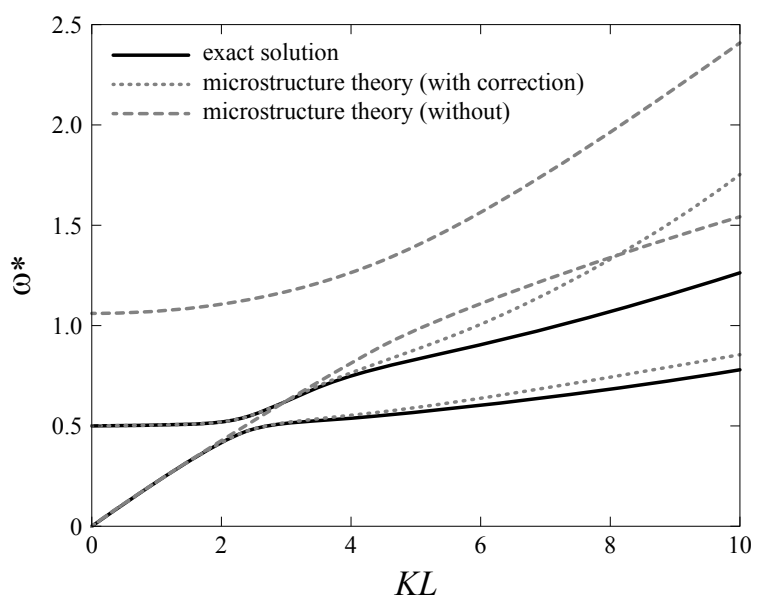

Figure 3. Comparison of the longitudinal dispersive curves with and without the correction factors. Adapted from [Huang and Sun 2008].

is optical (upper branch). Such a situation is noticed in many studies [Erofeyev 2003; Engelbrecht et al. 2005; Huang and Sun 2008; Berezovski et al. 2011a]. (As an example, the dispersion curves from the paper by Huang and Sun [2008] are shown in Figure 3.)

Direct calculations by the finite element method also display the changes in velocities due to dispersive effects [Gonella et al. 2011; Greene et al. 2012].

No band gaps are observed in the dispersion curves shown in Figures 2, 3. However, the existence of such band gaps has been demonstrated by Madeo et al. [2013] for unidirectional wave propagation in relaxed micromorphic media [Neff et al. 2014].

5.2. Phase and group velocities. The phase $\left(c_{\mathrm{ph}}=\omega / k\right)$ and group $\left(c_{\mathrm{gr}}=\partial \omega / \partial k\right)$ speeds corresponding to relation (64) are depicted in Figure 4. It must be noted that the asymptotic value of the acoustic phase speed approaches the value $c_{1} / c$ monotonically, while the group speed changes faster and nonmonotonically. It is possible to determine the dimensionless parameters which govern the process as [Engelbrecht et al. 2013]

$$
\gamma_{A}^{2}=c_{A}^{2} / c^{2}, \quad \gamma_{1}^{2}=c_{1}^{2} / c^{2}, \quad \Gamma=1-\gamma_{A}^{2}-\gamma_{1}^{2} .
$$

The parameter $\Gamma$ is crucial for distinction between dispersion types. If $\Gamma \geq 0$ then the dispersion is normal $\left(c_{\mathrm{gr}}<c_{\mathrm{ph}}\right)$ and if $\Gamma<0$ then the dispersion is anomalous $\left(c_{\mathrm{gr}}>c_{\mathrm{ph}}\right)$. In the dispersionless case $\Gamma=0$. Following Papargyri-Beskou et al. [2009] (Figure 5), these conditions are related to the ratio $h / g$ : if $h / g>1$ then the dispersion is normal, and if $h / g<1$ then the dispersion is anomalous. 


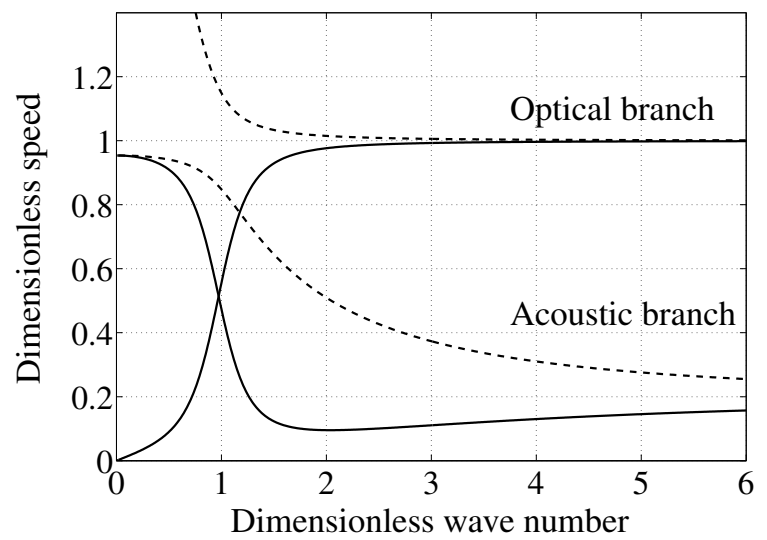

Figure 4. Group (solid line) and phase (dashed line) speed curves against the wave number, $c_{A}=0.3 c, c_{1}=0.2 c$.

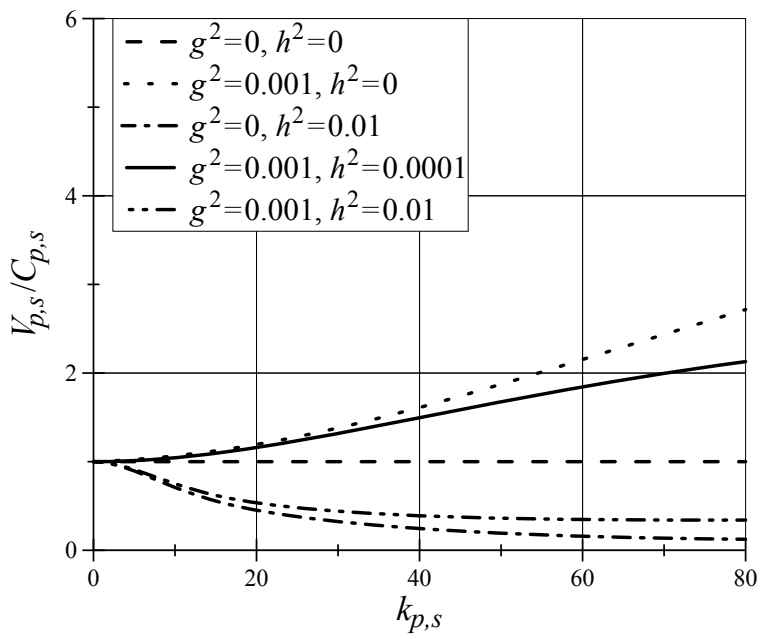

Figure 5. Dispersion curves of the $V_{p, s} / C_{p, s}$ versus $k_{p, s}$ type for elastic medium with microstructure obeying Equation (63). Adapted from [Papargyri-Beskou et al. 2009].

The parameter $\gamma_{A}$ is related to coupling effects, and it defines the dimensionless speed of long waves. The greater the value of $\gamma_{A}$, the smaller the speed of long waves. The parameter $\gamma_{1}$ is actually the ratio of speeds in macro- and microstructures. The greater the value of $\gamma_{1}$, the greater the speeds of short waves. The parameters $\gamma_{A}$ and $\gamma_{1}$ can be used for determining the differences between the full equation (33) and its asymptotic presentation (34); see [Peets et al. 2008]. In addition, a dimensionless parameter $\gamma_{A B}$ might be useful for asymptotic estimations, 


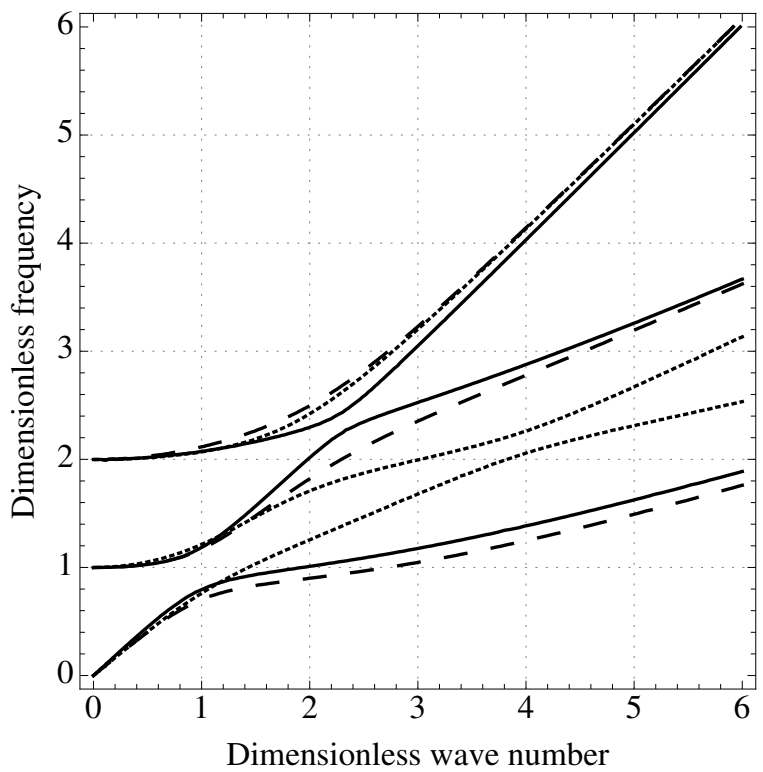

Figure 6. Comparison of dispersion curves of hierarchical microstructure model. Solid lines: Equation (43). Dashed lines: concurrent coupled microstructure model. Dotted lines: concurrent uncoupled microstructure model. Here $c_{A 1}=c_{A 2}=c_{A 12}=0.4 c$, $c_{1}=0.5 c, c_{1}=0.3 c$.

given by

$$
\gamma_{A B}^{2}=c_{A}^{2} / c_{B}^{2}=D^{2} I / \rho B^{2} L_{0}^{2}=\delta I^{*} D^{2} / B^{2},
$$

where it is assumed that $I=\rho l^{2} I^{*}, \delta=l^{2} / L_{0}^{2}$. As follows from (67), it involves the scale parameter $\delta$, which also gives weight to higher-order terms in the governing equations.

In the case of a hierarchical microstructure modelled by the system (36)-(38) or by (43), the dispersion relation reads as

$$
\begin{aligned}
& \left(c^{2} k^{2}-\omega^{2}\right)\left(c_{1} k^{2}-\omega^{2}+\omega_{1}^{2}\right)\left(c_{2}^{2} k^{2}-\omega^{2}+\omega_{2}^{2}\right) \\
& -c_{A 12}^{2} \omega_{2}^{2} k^{2}\left(c^{2} k^{2}-\omega^{2}\right)-c_{A 1}^{2} \omega_{1}^{2} k^{2}\left(c_{2}^{2}-\omega^{2}+\omega_{2}^{2}\right)=0 .
\end{aligned}
$$

Here, in addition to the notation of (43), we define $c_{A 12}^{2}=A_{12}^{2} / I_{1} B_{2}, \omega_{1}^{2}=1 / p_{1}^{2}$, and $\omega_{2}^{2}=1 / p_{2}^{2}$. The typical dispersion curves are shown in Figure 6 .

This model may involve an interesting physical phenomenon: negative group velocity (NGV), analysed by Peets et al. [2013]. Indeed, such a case is shown in Figure 7. The physical explanation for this phenomenon may be the following. It is known that the optical branches are related to nonpropagating oscillations 


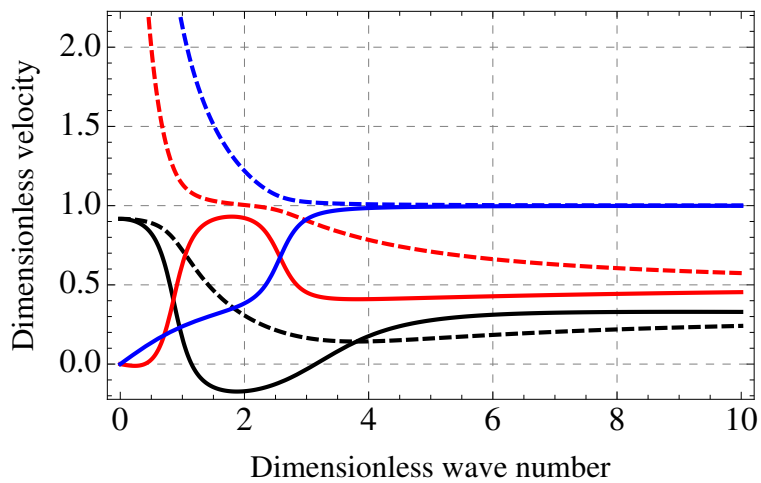

Figure 7. Group (solid line) and phase (dashed line) speed curves against the wave number, $c_{A}=0.3 c, c_{1}=0.2 c$.

[Brillouin 1946]. In the case shown in Figure 6, two optical branches can be very close to each other at certain frequencies. This can be considered as a preresonant situation: these nonpropagating oscillations are coupled, resulting in the NGV. This is also the reason for the multivalued phase velocity (Figure 7). Note that in optics the NGV is usually space-dependent, [Dogariu et al. 2001] but here, as seen from Figure 7, the NGV is dependent on the wave number. Consequently, for an arbitrary excitation with a wide spectrum, only some spectral components are affected.

5.3. Wave profiles. Dispersion effects are certainly reflected in wave profiles. A typical profile is shown in Figure 8 [Berezovski et al. 2013], where the influence of optical and acoustic branches of dispersion curves is seen. It is noted that high frequency oscillations due to the optical dispersion branch appear. A detailed analysis of such effects is presented by Tamm and Peets [2013]. Clearly, these effects must be taken into account not only in solving the direct problems but especially in nondestructive testing with acoustic waves. Several numerical results are presented for an impact-type excitation in [Askes and Metrikine 2002; Fish et al. 2002; Peets and Tamm 2010], for a triangular pulse in [Wang and Sun 2002], for a harmonic pulse in [Peets and Tamm 2010], for a burst-type pulse in [Greene et al. 2012].

5.4. Influence of nonlinearity. The nonlinear models described in Section 4 lead to Boussinesq-type equations. The possible balance of dispersion and nonlinearity may lead to soliton-type solutions. There are three essential problems related to solitons: (i) existence of solitons, (ii) emergence of solitons, and (iii) interaction of solitons. Contrary to the celebrated $\mathrm{KdV}$-solitons governed by an equation with first-order time derivative in a leading term, here the governing equations possess a second-order time derivative in leading terms. This means that, as in the classical wave equation, the waves propagate to the left and to the right (d'Alembert 

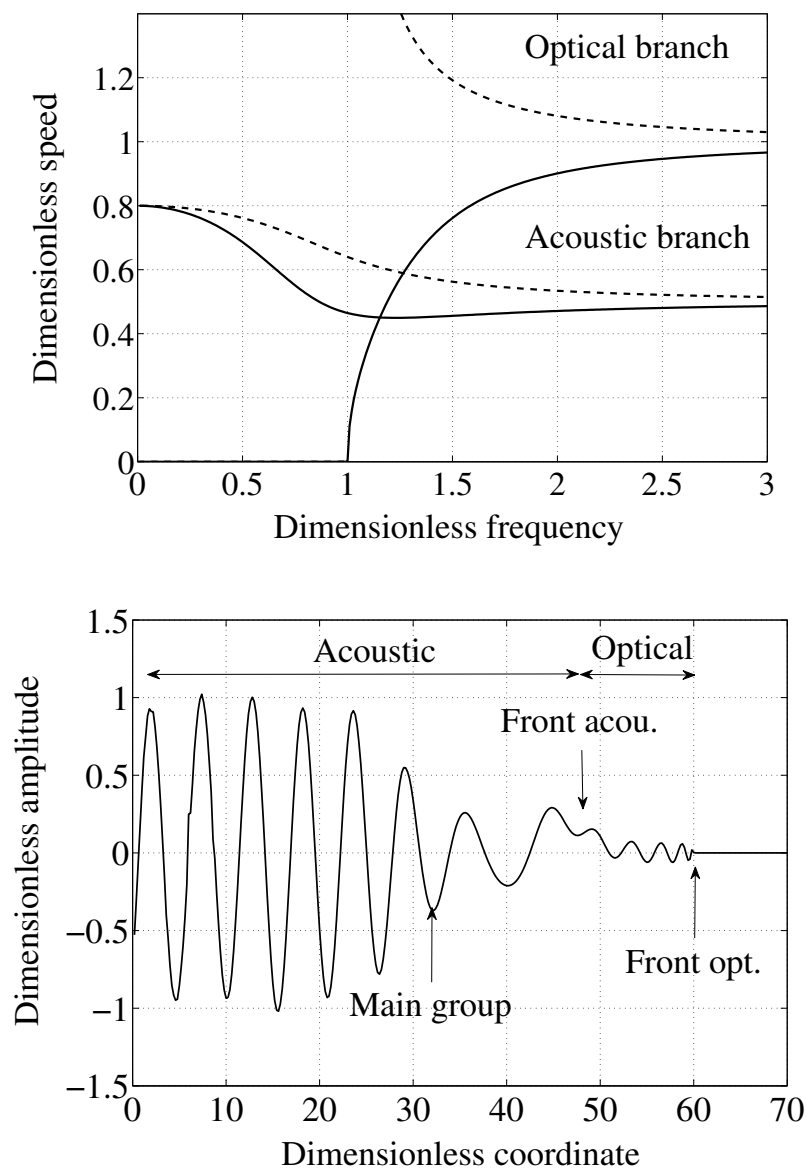

Figure 8. Top: phase (dotted lines) and group (solid lines) speed curves. Bottom: wave profile at 60 time steps. Here $c_{A}=0.6 c$, $c_{1}=0.5 c$, dimensionless frequency for the boundary condition is 0.8 .

solution), not in one direction, as results from the $\mathrm{KdV}$ equation. Most studies concerning solitons and the $\mathrm{KdV}$ equation focus on fluids. However, a historical review on solitons in elastic solids is presented in [Maugin 2011b]. Here we focus on models of nonlinear microstructured solids based on equations described in Section 4.

5.4.1. Existence of solitons. We take (48) as the basic equation. In its dimensionless form, it yields

$$
V_{T T}-b V_{X X}-\frac{1}{2} \mu\left(V^{2}\right)_{X X}=\delta\left(\beta V_{T T}-\gamma V_{X X}+\delta^{1 / 2} \frac{1}{2} \kappa\left(V_{X}^{2}\right)_{X}\right)_{X X},
$$

where $V=U_{X}, U=u / U_{0}, X=x / l, T=c t / l$ and, as before $\delta=l^{2} / \lambda^{2}$. The 
coefficients of (69) are related to the free energy function (44) by

$$
\begin{gathered}
b=1-\frac{D^{2}}{(\lambda+2 \mu) B}, \quad \mu=\frac{N U_{0}}{(\lambda+2 \mu) L_{0}}, \quad \beta=\frac{I D^{2}}{\rho l^{2} B^{2}}, \\
\gamma=\frac{C D^{2}}{(\lambda+2 \mu) B^{2} l^{2}}, \quad \kappa=\frac{D^{3} M U_{0}}{(\lambda+2 \mu) B^{3} l^{3} L_{0}} .
\end{gathered}
$$

The existence of a single solitary wave solution to (69) should satisfy the conditions [Janno and Engelbrecht 2005b]

$$
\begin{aligned}
& \frac{c_{s}^{2}-b}{\beta c_{s}^{2}-\gamma}>0, \quad\left(\frac{\beta c_{s}^{2}-\gamma}{c_{s}^{2}-b}\right)^{3}>\frac{4 \kappa}{\mu^{2}}, \\
& \mu \neq 0, \quad \beta c_{s}^{2}-\gamma \neq 0, \quad c_{s}^{2}-b \neq 0,
\end{aligned}
$$

where $c_{s}$ is a characteristic speed of solitary waves. For other types of governing equations, the solitary waves are described in [Maugin 1999; Erofeyev 2003; Porubov et al. 2009].

5.4.2. Emergence of solitons. In the classical example of the $\mathrm{KdV}$ equation, a harmonic initial condition leads to a train of solitons [Zabusky and Kruskal 1965]. Here the results should be two trains of solitons, propagating to the left and to the
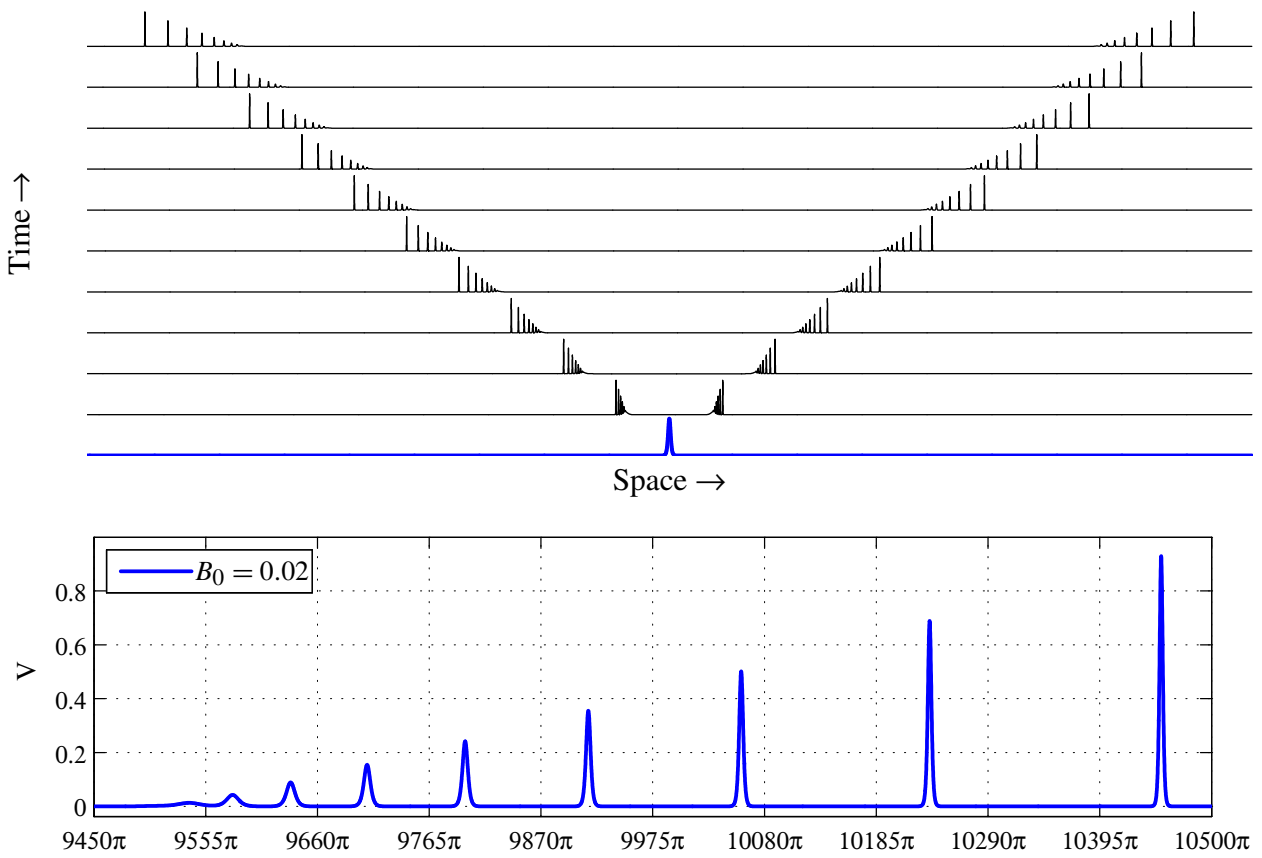

Figure 9. Formation of train of solitons for $B_{0}=0.02, K=5500$.

Profiles plotted every 1500 time steps. 
right. Indeed, it has been shown by numerical simulations [Engelbrecht et al. 2006; 2011] that solving (69) with an initial condition in the form of a single bell-type pulse results in two trains of solitons. Figure 9 shows the emergence process in the course of time. If dispersion is controlled by other higher-order derivatives, then the emergence process is somewhat different. Several examples are presented in [Maugin 1999]. Emergence of solitons is described also in laminates where the stress-strain law of layers is nonlinear and dispersion is caused by layering [Engelbrecht et al. 2007].

5.4.3. Interaction of solitary waves. This is a crucial problem when determining whether solitary waves behave like solitons or not. A soliton should interact with another soliton, keeping its amplitude (velocity), and only a phase change is allowed. This process has been intensively studied by many authors [Soerensen et al. 1984; Maugin and Christov 1997; Bogdan and Kosevich 1997; Maugin 1999; Christov et al. 2007; Salupere et al. 2008; Porubov 2009]. An intriguing question is the shape of solitary waves. Bearing in mind the microstructured solids, two nonlinearities are presented in (68): nonlinearity of the macrostructure and nonlinearity of the microstructure. It has been shown [Janno and Engelbrecht 2005b] that the existence of two nonlinearities leads to the asymmetry of a solitary wave. Note that, with $\kappa=0$ (no nonlinearity at the microstructure), (68) has the solitary wave solution [Porubov 2003; Janno and Engelbrecht 2005b]

$$
\begin{gathered}
V\left(X-c_{s} T\right)=A_{s} \operatorname{sech}^{2}\left[\frac{1}{2} \kappa_{1}\left(X-c_{s} T\right)\right], \\
A_{S}=3\left(c_{s}^{2}-b\right) / \mu, \quad \kappa_{1}^{2}=\left(c_{s}^{2}-b\right) / \delta\left(\beta c_{s}^{2}-\gamma\right) .
\end{gathered}
$$

This symmetric solution turns asymmetric if $\kappa \neq 0$ in (69). The evolution equation (one-wave equation) derived from (69) is a modified KdV-type equation, and for this an exact solution is found by Randrüüt and Braun [2010], which displays asymmetry due to the existence of the nonlinearity at the microstructure.

It is concluded by many authors that the interaction of solitary waves in such systems is usually accompanied by radiation [Christov et al. 2007; Salupere et al. 2008; Engelbrecht et al. 2011]. This means that the interaction can be considered elastic only in the course of several interactions, although the waves retain their individuality [Christov et al. 2007]. The governing equation may include more complicated nonlinearities, as in the case of chains of beads [Coste et al. 1997].

Remark 6. Turning again to waves in rods, the problems of emergence and interaction of solitary waves are studied in detail in [Porubov 2009].

\section{Thermal effects}

In Section 4, the governing equations were presented for microstructured thermoelastic solids. Definitely, the internal structure of thermoelastic solids displays not 


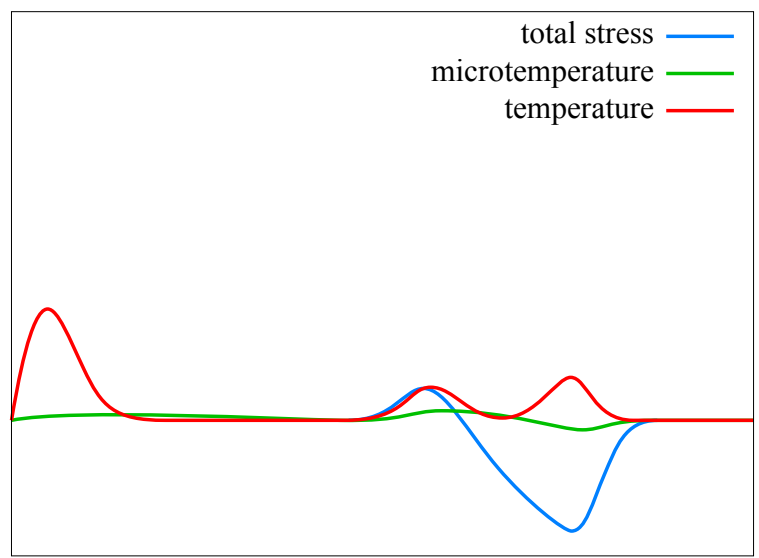

Figure 10. Distribution of temperature, stress, and microtemperature in a microstructured half-space at 350 time steps after thermal impact.

only differences in elastic properties but also in thermal characteristics. The internal temperature fluctuations which might be called microtemperature display a specific behaviour [Berezovski and Berezovski 2013; Berezovski and Engelbrecht 2013]. As an example, the propagation of a thermal pulse described by (52)-(54) is illustrated in Figure 10. Besides the usual diffusion of the macrotemperature $\theta$ in course of time close to boundary, wave-type behaviour of the total temperature is observed following the deformation wave. This is possible because of coupling effects between microtemperature (governed by a hyperbolic operator in (53)), stress, and macrotemperature.

\section{Material identification}

Physical effects due to the microstructure of solids serve as signatures about the structure. Changes in velocities and wave profiles and/or their spectra can be used to solve the inverse problems; that means determining material constants from the analysis of changes. As said before, waves are carriers of information. The problem, however, is complicated because the number of material constants is high. For example, Equation (33), which is a basic model in our discussion, involves, beside the properties of the macrostructure (density and the elastic constants), the properties of the microstructure (constants $B, C, D, I)$ and of coupling (constants $A, A^{\prime}$ ). In terms of the wave equation, these constants are grouped for coefficients. The situation is even more complicated in multiscale models or thermoelastic models (Section 4).

One possible approach to determine the material constants is to start from homogenisation methods [Santosa and Symes 1991; Forest 1998; 1999; Fish et al. 
2002; Wang and Sun 2002]. Some constants are presented in [Erofeyev 2003]. More contemporary homogenisation methods are described in [Jänicke and Steeb 2012; Fish and Kuznetsov 2012]. On other hand, numerical methods can be combined with material characterisation [Gonella et al. 2011; Greene et al. 2012]. The latter approach is also supported by theoretical results [Neff 2005; Neff and Forest 2007].

Another approach is to solve an inverse problem: given the structure of a model and an initial excitation and results of measurements, one has to determine the coefficients of the model. This approach is widely used in nondestructive evaluation (NDE) of material properties. There are many methods of NDE [Hellier 2001]; here we limit ourselves only to the possibilities of ultrasound NDE, based on the usage of acoustic waves as carriers of information. A detailed description of solving the inverse problem for the Mindlin-type micromorphic model is given in [Janno and Engelbrecht 2011]. The basic model is either the linear equation (34) or its nonlinear modification (48), together with their basic systems of two equations, like the system (20)-(21). The conditions for the existence of the solution together with its uniqueness and stability guarantee a well-posed problem. Harmonic waves and wave packets are used in the case of linear problems, and solitons in the case of nonlinear problems. The main ideas are to use changes in phase velocities [Janno and Engelbrecht 2005c] and properties of solitary waves [Janno and Engelbrecht 2005a] for determining material constants. Harmonic waves and Gaussian wave packets are used in the linear case. In the nonlinear case, a novel method is proposed based on measuring the asymmetry of a solitary wave [Janno and Engelbrecht 2005a]. The crucial point is to establish a number of possible coefficients which can be determined from solving a corresponding well-posed inverse problem.

\section{Discussion and final remarks}

Technological demands for microstructured materials are high, and there is a growing need to predict the behaviour of such structures under high-intensity and highfrequency excitations. As shown above, there exists a general framework for building appropriate mathematical models in order to grasp microscopic-to-macroscopic relations in materials. One might say that the unifying goal of this framework is to understand better the bulk behaviour of matter which depends on microscopic constituents and their properties. In this context, there are several avenues of research.

The first avenue leads towards developing the theory of microstructured continua. It is reflected in many monographs [Eringen 1999; Capriz 1989; Maugin 1999; 2013] and overviews [Mariano 2002] together with a lot of research papers. Several subfields are important: modelling of elastic microstructures, modelling 
of coupled fields (thermo-, electro-, magnetoelasticity), modelling of dislocations and phase transitions, etc. In this context, multiscale problems which depend on the character of excitations (the ratio $L_{0} / l$ ) become more and more important, resulting in hierarchical models.

The second avenue could be described as casting the theory of microstructured continua into concrete mathematical models, i.e., into systems of equations or single equations. In wave dynamics, this means deriving the modified systems of wave equations with leading terms of the second-order partial derivatives together with higher-order terms. These equations may be built in the form of certain hierarchies [Whitham 1974]. In this overview we moved along this avenue, presenting the mathematical models and analysing the corresponding physical effects.

The third avenue is related to numerical simulations. Due to complicated mathematical models and several scales, numerical schemes need to be modified in order to describe multiscales or coupling effects (see, for example, [Mariano and Stazi 2005; Vernerey et al. 2007; Berezovski et al. 2008]). Here we also use numerical simulations to demonstrate the changes in wave profiles.

The fourth avenue should lead to experimental verification. There are not very many results in this important direction. The early experiments of Potapov and Rodyushkin [2001] demonstrated the existence of solitary waves. The studies [Coste et al. 1997; Porter et al. 2009] described experiments with solitary waves in chains of beads, which give a possibility to build tunable one-dimensional phononic materials [Daraio et al. 2006]. Clearly, much is expected in moving along this avenue - not only phononic materials, but the general applications in the NDE should be further developed for more precise materials characterisation.

As said before, in this overview, attention was focussed on models of waves in their simplest one-dimensional setting. The concept of internal variables permits easy derivation of the mathematical models accounting for various physical effects. It is demonstrated that, beside the classical wave equation, the modified wave equations with higher-order terms form an interesting and challenging chapter in mathematical physics. These equations can be derived by satisfying thermodynamical constraints, and naturally involve both acoustic and optical branches in dispersion relations. This is extremely important in order to model the physical situation correctly (see, e.g., Figure 8).

The dispersion analysis reflects rich physical phenomena due to microstructure(s), and accounts for the multiscale problems, resulting in hierarchical equations. If nonlinear effects are included then the governing equations are of the Boussinesq type. The coupling of the macro- and microstructure leads to changes in velocities and wave profiles. The main conclusion from the analysis above is the following. The influence of the microstructure on wave propagation in solids 
is modelled best if (i) microinertia is taken into account, and (ii) the corresponding dispersion relation includes both acoustic (in-phase) and optical (out-of-phase) branches. If, however, some asymptotic procedures are applied in order to simplify the full model, then the simplified model should in some sense grasp the influence of the optical branch.

The classical wave equation is a cornerstone in mechanics and mathematical physics. It describes the propagation of an excitation in homogeneous elastic media. What is discussed above is how to modify this beautiful mathematical model in order to come closer to reality. As a result, the mathematical models involve higherorder derivatives and nonlinear terms which stem from the properties of materials. Such models gain more and more attention in contemporary engineering problems of dynamical response of materials and constructions.

It must be noted that the existence of a microstructure in a bulk material means that the constituents interact with each other and influence the macrobehaviour. This is a typical problem of complexity, where the behaviour of constituents leads to changes in the global behaviour [Nicolis and Nicolis 2007]. There are also attempts to cast the analysis into this pattern in multiscale materials [Engelbrecht 2009; Liu et al. 2010; Engelbrecht and Pastrone 2011]. However, such an approach is not used for describing solids only, it is also noted in fluids (see [Engelbrecht et al. 2010]).

Finally, the ideas worked out theoretically during the last century [Maugin 2013] have matured now to reach practical applications. The search certainly goes on, as said by Mariano [2012]:

... effective developments in applied sciences rely on a deep comprehension and command of the inner nature of the models involved and techniques utilised.

\section{References}

[Achenbach et al. 1968] J. D. Achenbach, C.-T. Sun, and G. Herrmann, "On the vibrations of a laminated body", J. Appl. Mech. (ASME) 35:4 (1968), 689-696.

[Andrianov et al. 2010] I. V. Andrianov, J. Awrejcewicz, and D. Weichert, "Improved continuous models for discrete media", Math. Probl. Eng. 2010 (2010), Article ID 986242.

[Andrianov et al. 2011] I. V. Andrianov, V. V. Danishevs'kyy, H. Topol, and D. Weichert, "Homogenization of a 1D nonlinear dynamical problem for periodic composites", Z. Angew. Math. Mech. 91:6 (2011), 523-534.

[Andrianov et al. 2013] I. V. Andrianov, V. V. Danishevs'kyy, O. I. Ryzhkov, and D. Weichert, “Dynamic homogenization and wave propagation in a nonlinear 1D composite material", Wave Motion 50:2 (2013), 271-281.

[Askar 1986] A. Askar, Lattice dynamical foundations of continuum theories: elasticity, piezoelectricity, viscoelasticity, plasticity, Theoretical and Applied Mechanics 2, World Scientific, Philadelphia, 1986. 
[Askes and Aifantis 2006] H. Askes and E. C. Aifantis, "Gradient elasticity theories in statics and dynamics: a unification of approaches", Int. J. Fract. 139:2 (2006), 297-304.

[Askes and Metrikine 2002] H. Askes and A. V. Metrikine, "One-dimensional dynamically consistent gradient elasticity models derived from a discrete microstructure, 2: Static and dynamic response", Eur. J. Mech. A Solids 21:4 (2002), 573-588.

[Askes and Metrikine 2005] H. Askes and A. V. Metrikine, "Higher-order continua derived from discrete media: continualisation aspects and boundary conditions", Int. J. Solids Struct. 42:1 (2005), 187-202.

[Askes et al. 2008] H. Askes, A. V. Metrikine, A. V. Pichugin, and T. Bennett, "Four simplified gradient elasticity models for the simulation of dispersive wave propagation", Philos. Mag. 88:2829 (2008), 3415-3443.

[Berezovski and Berezovski 2013] A. Berezovski and M. Berezovski, "Influence of microstructure on thermoelastic wave propagation", Acta Mech. 224:11 (2013), 2623-2633.

[Berezovski and Engelbrecht 2013] A. Berezovski and J. Engelbrecht, "Thermoelastic waves in microstructured solids: dual internal variables approach", J. Coupled Syst. Multiscale Dyn. 1:1 (2013), $112-119$.

[Berezovski et al. 2008] A. Berezovski, J. Engelbrecht, and G. A. Maugin, Numerical simulation of waves and fronts in inhomogeneous solids, World Scientific Series on Nonlinear Science Series A 62, World Scientific, Hackensack, NJ, 2008.

[Berezovski et al. 2010] A. Berezovski, J. Engelbrecht, and T. Peets, "Multiscale modeling of microstructured solids", Mech. Res. Commun. 37:6 (2010), 531-534.

[Berezovski et al. 2011a] A. Berezovski, J. Engelbrecht, and M. Berezovski, "Waves in microstructured solids: a unified viewpoint of modeling", Acta Mech. 220:1-4 (2011), 349-363.

[Berezovski et al. 2011b] A. Berezovski, J. Engelbrecht, and G. A. Maugin, "Generalized thermomechanics with dual internal variables", Arch. Appl. Mech. 81:2 (2011), 229-240.

[Berezovski et al. 2013] A. Berezovski, J. Engelbrecht, A. Salupere, K. Tamm, T. Peets, and M. Berezovski, "Dispersive waves in microstructured solids", Int. J. Solids Struct. 50:11-12 (2013), 1981-1990.

[Berezovski et al. 2014] A. Berezovski, J. Engelbrecht, and P. Ván, "Weakly nonlocal thermoelasticity for microstructured solids: microdeformation and microtemperature", Arch. Appl. Mech. 84:9-11 (2014), 1249-1261.

[Birman and Byrd 2007] V. Birman and L. W. Byrd, "Modeling and analysis of functionally graded materials and structures", Appl. Mech. Rev. (ASME) 60:5 (2007), 195-216.

[Bogdan and Kosevich 1997] M. Bogdan and A. Kosevich, "Interaction of moving solitons in a dispersive medium and regimes of their radiationless motion", Proc. Estonian Acad. Sci. Phys. Math. 46:1-2 (1997), 14-23.

[Born and von Kármán 1912] M. Born and T. von Kármán, "Über Schwingungen in Raumgittern”, Physik. Z. 13 (1912), 297-309.

[Brillouin 1946] L. Brillouin, Wave propagation in periodic structures: electric filters and crystal lattices, McGraw-Hill, New York, 1946.

[Capriz 1989] G. Capriz, Continua with microstructure, Springer Tracts in Natural Philosophy 35, Springer, New York, 1989.

[Casasso and Pastrone 2010] A. Casasso and F. Pastrone, "Wave propagation in solids with vectorial microstructures", Wave Motion 47:6 (2010), 358-369. 
[Challamel et al. 2009] N. Challamel, L. Rakotomanana, and L. Le Marrec, "A dispersive wave equation using nonlocal elasticity”, C. R. Mécanique 337:8 (2009), 591-595.

[Chen and Lee 2003] Y. Chen and J. D. Lee, "Connecting molecular dynamics to micromorphic theory, II: Balance laws”, Physica A 322 (2003), 377-392.

[Chen et al. 2003] Y. Chen, J. D. Lee, and A. Eskandarian, "Examining the physical foundation of continuum theories from the viewpoint of phonon dispersion relation", Int. J. Eng. Sci. 41:1 (2003), $61-83$.

[Chen et al. 2004] Y. Chen, J. D. Lee, and A. Eskandarian, "Atomistic viewpoint of the applicability of microcontinuum theories", Int. J. Solids Struct. 41:8 (2004), 2085-2097.

[Christov et al. 1996] C. I. Christov, G. A. Maugin, and M. G. Velarde, "Well-posed Boussinesq paradigm with purely spatial higher-order derivatives", Phys. Rev. E 54:4 (1996), 3621-3638.

[Christov et al. 2007] C. I. Christov, G. A. Maugin, and A. V. Porubov, "On Boussinesq's paradigm in nonlinear wave propagation”, C. R. Mécanique 335:9-10 (2007), 521-535.

[Cosserat and Cosserat 1909] E. Cosserat and F. Cosserat, Théorie des corps déformables, Hermann, Paris, 1909.

[Coste et al. 1997] C. Coste, E. Falcon, and S. Fauve, "Solitary waves in a chain of beads under Hertz contact”, Phys. Rev. E 56:5 (1997), 6104-6117.

[Craster et al. 2010] R. V. Craster, J. Kaplunov, and A. V. Pichugin, "High-frequency homogenization for periodic media", Proc. R. Soc. Lond. A 466:2120 (2010), 2341-2362.

[Curtin and Miller 2003] W. A. Curtin and R. E. Miller, "Atomistic/continuum coupling in computational materials science”, Model. Simul. Mater. Sci. Eng. 11:3 (2003), R33-R68.

[Daraio et al. 2006] C. Daraio, V. F. Nesterenko, E. B. Herbold, and S. Jin, “Tunability of solitary wave properties in one-dimensional strongly nonlinear phononic crystals", Phys. Rev. E 73:2 (2006), Article ID 026610.

[dell'Isola et al. 2009] F. dell'Isola, G. Sciarra, and S. Vidoli, "Generalized Hooke's law for isotropic second gradient materials", Proc. R. Soc. Lond. A 465:2107 (2009), 2177-2196.

[dell'Isola et al. 2012] F. dell'Isola, A. Madeo, and L. Placidi, "Linear plane wave propagation and normal transmission and reflection at discontinuity surfaces in second gradient 3D continua", $Z$. Angew. Math. Mech. 92:1 (2012), 52-71.

[Dogariu et al. 2001] A. Dogariu, A. Kuzmich, and L. J. Wang, "Transparent anomalous dispersion and superluminal light-pulse propagation at a negative group velocity", Phys. Rev. A 63:5 (2001), Article ID 053806.

[Duhem 1911a] P. M. M. Duhem, Traité d'énergétique ou de thermodynamique générale, vol. 1, Gauthier-Villars, Paris, 1911.

[Duhem 1911b] P. M. M. Duhem, Traité d'énergétique ou de thermodynamique générale, vol. 2, Gauthier-Villars, Paris, 1911.

[Engelbrecht 1997] J. Engelbrecht, Nonlinear wave dynamics: complexity and simplicity, Kluwer Texts in the Mathematical Sciences 17, Kluwer, Dordrecht, 1997.

[Engelbrecht 2009] J. Engelbrecht, "Complexity in mechanics", Rend. Sem. Mat. Univ. Politec. Torino 67:3 (2009), 293-325.

[Engelbrecht and Berezovski 2012] J. Engelbrecht and A. Berezovski, "Internal structures and internal variables in solids", J. Mech. Mater. Struct. 7:10 (2012), 983-996.

[Engelbrecht and Braun 1998] J. Engelbrecht and M. Braun, "Nonlinear waves in nonlocal media", Appl. Mech. Rev. (ASME) 51:8 (1998), 475-488. 
[Engelbrecht and Pastrone 2003] J. Engelbrecht and F. Pastrone, "Waves in microstructured solids with nonlinearities in microscale", Proc. Estonian Acad. Sci. Phys. Math. 52:1 (2003), 12-20.

[Engelbrecht and Pastrone 2011] J. Engelbrecht and F. Pastrone, "Non linear waves in complex microstructured solids", Mem. Accad. Sci. Torino Cl. Sci. Fis. Mat. Nat. (5) 35 (2011), 23-36.

[Engelbrecht and Salupere 2014] J. Engelbrecht and A. Salupere, "Scaling and hierarchies of wave motion in solids", Z. Angew. Math. Mech. 94:9 (2014), 775-783.

[Engelbrecht et al. 2005] J. Engelbrecht, A. Berezovski, F. Pastrone, and M. Braun, "Waves in microstructured materials and dispersion”, Philos. Mag. 85:33-35 (2005), 4127-4141.

[Engelbrecht et al. 2006] J. Engelbrecht, F. Pastrone, M. Braun, and A. Berezovski, "Hierarchies of waves in nonclassical materials", pp. 29-47 in Universality of nonclassical nonlinearity, edited by P. P. Delsanto, Springer, New York, 2006.

[Engelbrecht et al. 2007] J. Engelbrecht, A. Berezovski, and A. Salupere, "Nonlinear deformation waves in solids and dispersion", Wave Motion 44:6 (2007), 493-500.

[Engelbrecht et al. 2010] J. Engelbrecht, A. Berezovski, and T. Soomere, "Highlights in the research into complexity of nonlinear waves", Proc. Estonian Acad. Sci. Phys. Math. 59:2 (2010), 61-65.

[Engelbrecht et al. 2011] J. Engelbrecht, A. Salupere, and K. Tamm, "Waves in microstructured solids and the Boussinesq paradigm", Wave Motion 48:8 (2011), 717-726.

[Engelbrecht et al. 2013] J. Engelbrecht, T. Peets, K. Tamm, and A. Salupere, "Deformation waves in microstructured solids and dimensionless parameters", Proc. Estonian Acad. Sci. Phys. Math. 62:2 (2013), 109-115.

[Eringen 1962] A. C. Eringen, Nonlinear theory of continuous media, McGraw-Hill, New York, 1962.

[Eringen 1964] A. C. Eringen, "Simple microfluids", Int. J. Eng. Sci. 2:2 (1964), 205-217.

[Eringen 1969] A. C. Eringen, "Micropolar fluids with stretch", Int. J. Eng. Sci. 7:1 (1969), 115-127.

[Eringen 1972] A. C. Eringen, "Linear theory of nonlocal elasticity and dispersion of plane waves", Int. J. Eng. Sci. 10:5 (1972), 425-435.

[Eringen 1999] A. C. Eringen, Microcontinuum field theories, I: Foundations and solids, Springer, New York, 1999.

[Eringen and Suhubi 1964a] A. C. Eringen and E. S. Suhubi, "Nonlinear theory of simple microelastic solids, I", Int. J. Eng. Sci. 2:2 (1964), 189-203.

[Eringen and Suhubi 1964b] A. C. Eringen and E. S. Suhubi, "Nonlinear theory of simple microelastic solids, II", Int. J. Eng. Sci. 2:4 (1964), 389-404.

[Erofeyev 2003] V. I. Erofeyev, Wave processes in solids with microstructure, Stability, Vibration and Control of Systems, Series A 8, World Scientific, Singapore, 2003.

[Fish and Kuznetsov 2012] J. Fish and S. Kuznetsov, "From homogenization to generalized continua”, Int. J. Comput. Methods Eng. Sci. Mech. 13:2 (2012), 77-87.

[Fish et al. 2002] J. Fish, W. Chen, and G. Nagai, "Non-local dispersive model for wave propagation in heterogeneous media: one-dimensional case", Int. J. Numer. Methods Eng. 54:3 (2002), 331-346.

[Fish et al. 2005] J. Fish, W. Chen, and Y. Tang, "Generalized mathematical homogenization of atomistic media at finite temperatures", Int. J. Multiscale Comput. Eng. 3:4 (2005), 393-413.

[Forest 1998] S. Forest, "Mechanics of generalized continua: construction by homogenizaton", $J$. Phys. (France) IV 8:PR4 (1998), 39-48.

[Forest 1999] S. Forest, "Homogenization methods and the mechanics of generalized continua", pp. 35-48 in Geometry, continua and microstructure (Paris, 1997), edited by G. A. Maugin, Travaux en Cours 60, Hermann, Paris, 1999. 
[Gates et al. 2005] T. S. Gates, G. M. Odegard, S. J. V. Frankland, and T. C. Clancy, "Computational materials: multi-scale modeling and simulation of nanostructured materials", Compos. Sci. Technol. 65:15-16 (2005), 2416-2434.

[Germain 1973] P. Germain, "The method of virtual power in continuum mechanics, 2: Microstructure”, SIAM J. Appl. Math. 25:3 (1973), 556-575.

[Goddard 1986] J. D. Goddard, "Microstructural origins of continuum stress fields: a brief history and some unresolved issues", pp. 179-208 in Recent developments in structured continua (Windsor, ON, 1985), edited by D. De Kee and P. N. Kaloni, Pitman Res. Notes Math. Ser. 143, Longman, Harlow, 1986.

[Gonella et al. 2011] S. Gonella, M. S. Greene, and W. K. Liu, "Characterization of heterogeneous solids via wave methods in computational microelasticity", J. Mech. Phys. Solids 59:5 (2011), 959974.

[Greene et al. 2012] M. S. Greene, S. Gonella, and W. K. Liu, "Microelastic wave field signatures and their implications for microstructure identification", Int. J. Solids Struct. 49:22 (2012), 31483157.

[Hellier 2001] C. J. Hellier, Handbook of nondestructive evaluation, McGraw-Hill, New York, 2001.

[Huang and Sun 2008] G. L. Huang and C.-T. Sun, "A higher-order continuum model for elastic media with multiphased microstructure", Mech. Adv. Mater. Struct. 15:8 (2008), 550-557.

[Jakata and Every 2008] K. Jakata and A. G. Every, "Determination of the dispersive elastic constants of the cubic crystals Ge, Si, GaAs, and InSb”, Phys. Rev. B 77:17 (2008), Article ID 174301.

[Jänicke and Steeb 2012] R. Jänicke and H. Steeb, "Wave propagation in periodic microstructures by homogenisation of extended continua”, Comput. Mater. Sci. 52:1 (2012), 209-211.

[Janno and Engelbrecht 2005a] J. Janno and J. Engelbrecht, "An inverse solitary wave problem related to microstructured materials”, Inverse Probl. 21:6 (2005), 2019-2034.

[Janno and Engelbrecht 2005b] J. Janno and J. Engelbrecht, "Solitary waves in nonlinear microstructured materials", J. Phys. A 38:23 (2005), 5159-5172.

[Janno and Engelbrecht 2005c] J. Janno and J. Engelbrecht, "Waves in microstructured solids: inverse problems", Wave Motion 43:1 (2005), 1-11.

[Janno and Engelbrecht 2011] J. Janno and J. Engelbrecht, Microstructured materials: inverse problems, Springer, Heidelberg, 2011.

[Kirchner and Steinmann 2004] N. Kirchner and P. Steinmann, "Modeling microstructured materials: a comparison of gradient and micromorphic continua", pp. 35-42 in Modelling of cohesivefrictional materials, edited by H. J. Herrmann et al., Taylor and Francis, London, 2004.

[Kröner 1968] E. Kröner, "Interrelations between various branches of continuum mechanics", pp. 330-340 in Mechanics of generalized continua (Freudenstadt and Stuttgart, 1967), edited by E. Kröner, Springer, Berlin, 1968.

[Kunin 1975] I. A. Kunin, Теория упругих сред с микроструктурой, Nauka, Moscow, 1975. Translated as Elastic media with microstructure, I: One-dimensional models, Springer Series in Solid-State Sciences 26, Springer, Berlin, 1982.

[Liu et al. 2010] W. K. Liu, D. Qian, S. Gonella, S. Li, W. Chen, and S. Chirputkar, "Multiscale methods for mechanical science of complex materials: bridging from quantum to stochastic multiresolution continuum", Int. J. Numer. Methods Eng. 83:8-9 (2010), 1039-1080.

[Love 1944] A. E. H. Love, A treatise on the mathematical theory of elasticity, 4th ed., Dover, New York, 1944. 
[Madeo et al. 2013] A. Madeo, P. Neff, I.-D. Ghiba, L. Placidi, and G. Rosi, "Wave propagation in relaxed micromorphic continua: modeling metamaterials with frequency band-gaps", Contin. Mech. Therm. (2013), 1-20. In press.

[Mahamood et al. 2012] R. M. Mahamood, E. T. Akinlabi, M. Shukla, and S. Pityana, "Functionally graded material: an overview", pp. 1593-1597 in Proceedings of the World Congress on Engineering (London, 2012), vol. III, edited by S. I. Ao et al., Lecture Notes in Engineering and Computer Science 2199, Newswood, Hong Kong, 2012.

[Maranganti and Sharma 2007] R. Maranganti and P. Sharma, "A novel atomistic approach to determine strain-gradient elasticity constants: tabulation and comparison for various metals, semiconductors, silica, polymers and the (lr) relevance for nanotechnologies", J. Mech. Phys. Solids 55:9 (2007), 1823-1852.

[Mariano 2002] P. M. Mariano, "Multifield theories in mechanics of solids", pp. 1-93 Advances in Applied Mechanics 38, Elsevier, New York, 2002.

[Mariano 2012] P. M. Mariano, "Perspectives in continuum mechanics: a preface", Math. Methods Appl. Sci. 35:15 (2012), 1737-1740.

[Mariano and Stazi 2005] P. M. Mariano and F. L. Stazi, "Computational aspects of the mechanics of complex materials", Arch. Comput. Methods Eng. 12:4 (2005), 391-478.

[Maugin 1993] G. A. Maugin, Material inhomogeneities in elasticity, Applied Mathematics and Mathematical Computation 3, Chapman and Hall, London, 1993.

[Maugin 1995] G. A. Maugin, "On some generalizations of Boussinesq and KdV systems", Proc. Estonian Acad. Sci. Phys. Math. 44:1 (1995), 40-55.

[Maugin 1999] G. A. Maugin, Nonlinear waves in elastic crystals, Oxford University Press, 1999.

[Maugin 2006] G. A. Maugin, "On the thermomechanics of continuous media with diffusion and/or weak nonlocality”, Arch. Appl. Mech. 75:10-12 (2006), 723-738.

[Maugin 2011a] G. A. Maugin, Configurational forces: thermomechanics, physics, mathematics, and numerics, CRC, Boca Raton, FL, 2011.

[Maugin 2011b] G. A. Maugin, "Solitons in elastic solids (1938-2010)", Mech. Res. Commun. 38:5 (2011), 341-349.

[Maugin 2013] G. A. Maugin, Continuum mechanics through the twentieth century: a concise historical perspective, Solid Mechanics and its Applications 196, Springer, Dordrecht, 2013.

[Maugin and Christov 1997] G. A. Maugin and C. I. Christov, "Nonlinear duality between elastic waves and quasi-particles in microstructured solids", Proc. Estonian Acad. Sci. Phys. Math. 46:1-2 (1997), 78-84.

[Maugin and Muschik 1994] G. A. Maugin and W. Muschik, "Thermodynamics with internal variables, I: General concepts", J. Non Equilib. Thermodyn. 19:3 (1994), 217-249.

[Metrikine 2006] A. V. Metrikine, "On causality of the gradient elasticity models", J. Sound Vib. 297:3-5 (2006), 727-742.

[Metrikine and Askes 2002] A. V. Metrikine and H. Askes, "One-dimensional dynamically consistent gradient elasticity models derived from a discrete microstructure, 1: Generic formulation", Eur. J. Mech. A Solids 21:4 (2002), 555-572.

[Mindlin 1964] R. D. Mindlin, "Micro-structure in linear elasticity", Arch. Ration. Mech. Anal. 16 (1964), 51-78.

[Murdoch 2010] A. I. Murdoch, "On molecular modelling and continuum concepts", J. Elasticity 100:1-2 (2010), 33-61. 
[Neff 2005] P. Neff, "On material constants for micromorphic continua", pp. 337-348 in Trends in applications of mathematics to mechanics (Seeheim, 2004), edited by Y. Wang and K. Hutter, Shaker, Aachen, 2005.

[Neff 2006] P. Neff, "A finite-strain elastic-plastic Cosserat theory for polycrystals with grain rotations”, Int. J. Eng. Sci. 44:8-9 (2006), 574-594.

[Neff and Forest 2007] P. Neff and S. Forest, "A geometrically exact micromorphic model for elastic metallic foams accounting for affine microstructure. Modelling, existence of minimizers, identification of moduli and computational results", J. Elasticity 87:2-3 (2007), 239-276.

[Neff and Jeong 2009] P. Neff and J. Jeong, "A new paradigm: the linear isotropic Cosserat model with conformally invariant curvature energy”, Z. Angew. Math. Mech. 89:2 (2009), 107-122.

[Neff et al. 2014] P. Neff, I.-D. Ghiba, A. Madeo, L. Placidi, and G. Rosi, "A unifying perspective: the relaxed linear micromorphic continuum", Contin. Mech. Therm. 26:5 (2014), 639-681.

[Nicolis and Nicolis 2007] G. Nicolis and C. Nicolis, Foundations of complex systems: nonlinear dynamics, statistical physics, information and prediction, World Scientific, Hackensack, NJ, 2007.

[Nowacki 1972] W. Nowacki, Termosprężystość, Polish Scientific Publishers, Warsaw, 1972. Translated as Thermoelasticity, 2nd ed., Pergamon, Oxford, 1986.

[Papargyri-Beskou et al. 2009] S. Papargyri-Beskou, D. Polyzos, and D. E. Beskos, "Wave dispersion in gradient elastic solids and structures: a unified treatment”, Int. J. Solids Struct. 46:21 (2009), 3751-3759.

[Pastrone et al. 2004] F. Pastrone, P. Cermelli, and A. V. Porubov, "Nonlinear waves in 1-D solids with microstructure", Mater. Phys. Mech. 7:1 (2004), 9-16.

[Peets and Tamm 2010] T. Peets and K. Tamm, "Dispersion analysis of wave motion in microstructured solids", pp. 349-354 in IUTAM Symposium on Recent Advances of Acoustic Waves in Solids (Taipei, 2009), edited by T.-T. Wu and C.-C. Ma, IUTAM Bookseries 26, Springer, Netherlands, 2010.

[Peets et al. 2008] T. Peets, M. Randrüüt, and J. Engelbrecht, "On modelling dispersion in microstructured solids", Wave Motion 45:4 (2008), 471-480.

[Peets et al. 2013] T. Peets, D. Kartofelev, K. Tamm, and J. Engelbrecht, "Waves in microstructured solids and negative group velocity", Europhys. Lett. 103:1 (2013), Article ID 16001.

[Pichugin et al. 2008] A. V. Pichugin, H. Askes, and A. Tyas, "Asymptotic equivalence of homogenisation procedures and fine-tuning of continuum theories", J. Sound Vib. 313:3-5 (2008), 858-874.

[Polyzos and Fotiadis 2012] D. Polyzos and D. I. Fotiadis, "Derivation of Mindlin's first and second strain gradient elastic theory via simple lattice and continuum models", Int. J. Solids Struct. 49:3-4 (2012), 470-480.

[Porter et al. 2009] M. A. Porter, C. Daraio, I. Szelengowicz, E. B. Herbold, and P. G. Kevrekidis, "Highly nonlinear solitary waves in heterogeneous periodic granular media", Physica D 238:6 (2009), 666-676.

[Porubov 2000] A. V. Porubov, "Strain solitary waves in an elastic rod with microstructure", Rend. Sem. Mat. Univ. Politec. Torino 58:2 (2000), 189-198.

[Porubov 2003] A. V. Porubov, Amplification of nonlinear strain waves in solids, Stability, Vibration and Control of Systems, Series A 9, World Scientific, Singapore, 2003.

[Porubov 2009] A. V. Porubov, Локализация нелинейных волн деформации: асимптотические и численные методы исследования, Fizmatlit, Moscow, 2009. Translated as Localization of nonlinear waves: asymptotic and numerical methods of investigation. 
[Porubov and Maugin 2005] A. V. Porubov and G. A. Maugin, "Longitudinal strain solitary waves in presence of cubic non-linearity", Int. J. Non-Linear Mech. 40:7 (2005), 1041-1048.

[Porubov et al. 2009] A. V. Porubov, E. L. Aero, and G. A. Maugin, "Two approaches to study essentially nonlinear and dispersive properties of the internal structure of materials", Phys. Rev. E 79:4 (2009), Article ID 046608.

[Potapov and Rodyushkin 2001] A. I. Potapov and V. M. Rodyushkin, “Экспериментальное исследование волн деформации в материалах с микроструктурой”, Akust. Zh. 47:3 (2001), 407-412. Translated as "Experimental study of strain waves in materials with microstructure" in Acoust. Phys. 47:3 (2001), 347-352.

[Randrüüt and Braun 2010] M. Randrüüt and M. Braun, "On one-dimensional solitary waves in microstructured solids", Wave Motion 47:4 (2010), 217-230.

[Salupere et al. 2008] A. Salupere, K. Tamm, and J. Engelbrecht, "Numerical simulation of interaction of solitary deformation waves in microstructured solids", Int. J. Non-Linear Mech. 43:3 (2008), 201-208.

[Samsonov 2001] A. M. Samsonov, Strain solitons in solids and how to construct them, Chapman and Hall/CRC, Boca Raton, FL, 2001.

[Santosa and Symes 1991] F. Santosa and W. W. Symes, "A dispersive effective medium for wave propagation in periodic composites", SIAM J. Appl. Math. 51:4 (1991), 984-1005.

[Schrödinger 1914] E. Schrödinger, "Zur dynamik elastisch gekoppelter Punktsysteme", Ann. Phys. 349:14 (1914), 916-934.

[Seeger 2010] A. Seeger, "Historical note: on the simulation of dispersive wave propagation by elasticity models", Philos. Mag. 90:9 (2010), 1101-1104.

[Soerensen et al. 1984] M. P. Soerensen, P. L. Christiansen, and P. S. Lomdahl, "Solitary waves on nonlinear elastic rods, I", J. Acoust. Soc. Amer. 76:3 (1984), 871-879.

[Sun et al. 1968] C.-T. Sun, J. D. Achenbach, and G. Herrmann, "Continuum theory for a laminated medium", J. Appl. Mech. (ASME) 35:3 (1968), 467-475.

[Tamm and Peets 2013] K. Tamm and T. Peets, "On the influence of internal degrees of freedom on dispersion in microstructured solids", Mech. Res. Commun. 47 (2013), 106-111.

[Ván et al. 2008] P. Ván, A. Berezovski, and J. Engelbrecht, "Internal variables and dynamic degrees of freedom", J. Non Equilib. Thermodyn. 33:3 (2008), 235-254.

[Vernerey et al. 2007] F. Vernerey, W. K. Liu, and B. Moran, "Multi-scale micromorphic theory for hierarchical materials", J. Mech. Phys. Solids 55:12 (2007), 2603-2651.

[Wang and Sun 2002] Z.-P. Wang and C.-T. Sun, "Modeling micro-inertia in heterogeneous materials under dynamic loading”, Wave Motion 36:4 (2002), 473-485.

[Webb et al. 2008] E. B. Webb, III, J. A. Zimmerman, and S. C. Seel, "Reconsideration of continuum thermomechanical quantities in atomic scale simulations", Math. Mech. Solids 13:3-4 (2008), 221266.

[Whitham 1974] G. B. Whitham, Linear and nonlinear waves, Wiley, New York, 1974.

[Yin et al. 2004] H. M. Yin, L. Z. Sun, and G. H. Paulino, "Micromechanics-based elastic model for functionally graded materials with particle interactions", Acta Mater. 52:12 (2004), 3535-3543.

[Zabusky and Kruskal 1965] N. J. Zabusky and M. D. Kruskal, "Interaction of "solitons" in a collisionless plasma and the recurrence of initial states", Phys. Rev. Lett. 15:6 (1965), 240-243.

[Zeng et al. 2006] X. Zeng, Y. Chen, and J. D. Lee, "Determining material constants in nonlocal micromorphic theory through phonon dispersion relations", Int. J. Eng. Sci. 44:18-19 (2006), 13341345 . 
[Zhou 2005] M. Zhou, "Thermomechanical continuum representation of atomistic deformation at arbitrary size scales”, Proc. R. Soc. Lond. A 461:2063 (2005), 3437-3472.

[Ziegler 1977] F. Ziegler, "Wave propagation in periodic and disordered layered composite elastic materials”, Int. J. Solids Struct. 13:4 (1977), 293-305.

[Zimmerman et al. 2002] J. A. Zimmerman, R. E. Jones, P. A. Klein, D. J. Bammann, E. B. Webb, III, and J. J. Hoyt, "Continuum definitions for stress in atomistic simulation", Technical Report SAND2002-8608, Sandia National Laboratories, Livermore, CA and Albuquerque, NM, 2002.

Received 29 Oct 2013. Revised 16 May 2014. Accepted 21 Jul 2014.

JÜRI ENGELBRECHT: je@ioc.ee

Centre for Nonlinear Studies (CENS), Institute of Cybernetics at Tallinn University of Technology, Akadeemia Tee 21, 12618 Tallinn, Estonia

ARKADI BEREZOVSKI: arkadi.berezovski@cs.ioc.ee

Centre for Nonlinear Studies (CENS), Institute of Cybernetics at Tallinn University of Technology, Akadeemia Tee 21, 12618 Tallinn, Estonia

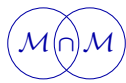

